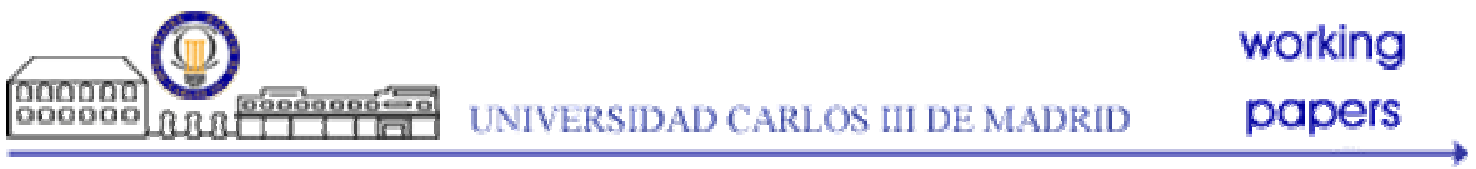

Working Paper 05-39

Statistics and Econometrics Series 06

June 2005
Departamento de Estadística

Universidad Carlos III de Madrid

Calle Madrid, 126

28903 Getafe (Spain)

Fax (34) 91 624-98-49

\title{
MARGINAL PRODUCTIVITY INDEX POLICIES FOR SCHEDULING A MULTICLASS DELAY-/LOSS-SENSITIVE QUEUE
}

\author{
José Niño-Mora *
}

\begin{abstract}
We address the problem of scheduling a multiclass $M / M / 1$ queue with a finite dedicated buffer for each class. Some classes are delay-sensitive, modeling real-time traffic (e.g. voice, video), whereas others are loss-sensitive, modeling nonreal-time traffic (e.g. data). Different levels of tolerance to delay and loss are modeled by appropriate linear holding cost and rejection cost rates. The goal is to design well-grounded and tractable scheduling policies which nearly minimize the discounted or long-run average expected cost objective. We develop new dynamic index policies, prescribing to give higher service priority to classes with larger index values, where the priority index of a class measures the marginal productivity of work at its current state. To construct the indices, we deploy the theory of marginal productivity indices (MPIs) and PCLindexability we have introduced in recent work, and further introduce significant extensions to such theory motivated by phenomena observed in the model of concern. The MPI policies are shown to furnish new, insightful structural results, and to exhibit a nearly optimal performance in a computational study.
\end{abstract}

Keywords: multiclass queues; control of queues; finite buffers; delay-sensitive; losssensitive; restless bandits; stochastic scheduling; index policies; indexability; control by price; Markov decision processes; marginal productivity; bias optimality

*Departamento de Estadística, Universidad Carlos III de Madrid, C/ Madrid 126, 28903 Getafe (Madrid), e-mail: jose.nino@uc3m.es. Research supported in part by the Spanish Ministry of Education and Science under a Ramón y Cajal Investigator Award and project MTM2004-02334, and by the European Union's Network of Excelence Euro-NGI. 


\section{Introduction}

Motivated by applications involving the dynamic control of heterogeneous traffic flows vying for access to service resources, e.g. in manufacturing or computer-communication systems, researchers have investigated in a variety of models the problem of finding a policy for dynamic scheduling of a multiclass queue which optimizes a performance objective of concern. Due to analytical tractability, most of such studies have addressed models where queues have infinite buffer space, so that the objective to optimize is a measure of average delay or congestion. Fruits of such work include the optimality of static index policies in a variety of settings. The earliest result in such vein concerns the optimal scheduling of a multiclass $M / M / 1$ queue, where traffic of class $k$ has arrival and service rates $\lambda_{k}$ and $\mu_{k}$, respectively, and accrues holding costs at rate $c_{k}$ per unit time per customer in system. It is proven in Cox and Smith [2] that the now classic $c \mu$-rule, which services at each decision epoch a nonempty class $k$ attaining the largest value of index $\nu_{k}=c_{k} \mu_{k}$, minimizes the (long-run) average cost rate.

In contrast, corresponding models where queues have finite buffer space have received remarkably scarce research attention, despite their obviously higher practical relevance. Finite buffers bring the possibility of customer losses, as arrivals find buffers full. This creates complicated boundary effects, which typically render such models intractable to analysis. Thus, e.g. in the variation on the above model where there is a finite dedicated buffer for each class, even the structure of the optimal policy remains elusive, being known only in exceptional cases.

The incorporation of linear holding cost rates $c_{k}$ and rejection cost rates $r_{k}$, along with finite buffers of size $n_{k}$ for each traffic class $k$, allows us to model different relative sensitivities to delays and losses. This is particularly relevant in models for modern computer-communication networks. Thus, traditional Internet traffic, such as e-mail and FTP, is primarily loss-sensitive, tolerating relatively long delays, and hence their requirements are accommodated by provisioning long buffers. In contrast, emerging Internet traffic, generated by interactive and multimedia applications such as IP telephony, video conferencing and networked games, is primarily delay-sensitive, having a higher relative tolerance for losses. Their requirements thus call for use of shorter buffers. This raises the issue of how to design tractable policies for dynamically scheduling a given mix of traffic types, consistently with performance objectives.

In this paper we investigate such issue in the setting of a multiclass $M / M / 1$ queue with finite dedicated buffers, under both discounted and average cost criteria. Before discussing our approach and results, we next briefly review the scarce previous work on such model. Milito and Levy [6] consider the pure loss-sensitive case where $r_{k}>0=c_{k}$ for each class $k$ in a two-class system, under the symmetry condition $\mu_{1}=\mu_{2}$. They show that a discount-optimal policy is characterized by a monotone increasing switching curve. Namely, if it is optimal to serve class 1 in state $\left(i_{1}, i_{2}\right)$ (joint queue length), then so it is to serve it in state $\left(i_{1}+1, i_{2}\right)$, and similarly for class 2. Sparaggis et al. [12] assume the stronger symmetry condition that rejection costs, arrival and service rates are each identical across classes (while the amount of buffer space may differ by class). They prove that the dynamic index rule which services at each decision epoch a nonempty class with the least number of empty buffer spaces minimizes the average loss rate. See also Wasserman and Bambos [13]. Kim and Van Oyen [4] extend Milito and Levy's [6] result to the case where the cost parameters of each of two classes $k$ satisfy the condition

$$
\alpha r_{k} \geq c_{k}
$$

where $\alpha>0$ is the exponential discount factor. Condition (1) will also play a central role in our results. It means that the cost of rejecting a customer $\left(r_{k}\right)$ is greater than or equal to the total discounted cost of holding it forever in the system $\left(c_{k} / \alpha\right)$. Kim and Van Oyen [4] further show by examples that, if condition (1) is violated, the optimal policy need not be given by a monotone increasing switching curve.

Our approach is based on viewing the model as a special case of the restless bandit problem. This concerns the optimal dynamic scheduling of a collection of stochastic projects, modeled as restless bandits, i.e. binary-action (work/rest) Markov decision processes (MDPs), which can change state under either action, and at most one of which can be engaged at a time. While the nonrestless case - where rested projects do not change state- is solved optimally by Gittins' [3] index policy, the restless case is, in general, intractable. Whittle [14] introduced an index for restless bandits, which reduces to Gittins' in the nonrestless case, and proposed as a heuristic the corresponding index policy: work at each time on a bandit with largest index value. However, the Whittle index only exists for a restricted class of bandits, termed indexable. In NiñoMora [7, 8, 10] we have developed a theory of indexability for restless bandits, which includes: the general concept of a bandit's marginal productivity index (MPI), which significantly extends the scope of the Whittle index; an intuitive characterization of indexable bandits as those obeying the economics law of diminishing marginal returns (to work), consistently with a family of threshold policies; sufficient indexability conditions, 
based on satisfaction of partial conservation laws (PCLs); and a one-pass index-computing algorithm. In short, a bandit's MPI measures the marginal value of work at each state. Thus, the MPI policy seeks to engage at each time a bandit where work is most productive, using the MPI as a proxy productivity measure. In this paper we both draw on such theory to obtain new MPI policies for scheduling a multiclass queue with finite dedicated buffers, and extend it to accommodate new phenomena observed in the model.

We next outline and discuss our results. Our analyses lead us to consider the following (not mutually exclusive) five types of traffic classes, relative to a discount factor $\alpha \geq 0$ : we say that a class $k$ is

- $\alpha$-discount loss-sensitive if $r_{k}>0$ and $\alpha r_{k} \geq c_{k}$;

- pure loss-sensitive if $r_{k}>0=c_{k}$;

- $\alpha$-discount delay-sensitive if $\alpha r_{k} \leq c_{k}>0$;

- delay-sensitive if $c_{k}>0$;

- pure delay-sensitive if $c_{k}>0=r_{k}$.

Consider an $\alpha$-discount loss-sensitive class $k$, with $\alpha>0$. We establish that it satisfies our $P C L$ indexability conditions, and give a recursion for calculating its discounted $M P I \nu_{k}^{\alpha, *}\left(i_{k}\right)$, as a function of the number of empty buffer spaces $i_{k}$, along with a strong characterization of the MPI as an optimal marginal productivity rate relative to active-state sets. The MPI $\nu_{k}^{\alpha, *}\left(i_{k}\right)$ is nonincreasing in $i_{k}$ and does not depend on the buffer size, consistently with the optimal least-empty-buffer-spaces rule in the symmetric case considered in Sparaggis et al. [12].

In the pure loss-sensitive case, we further consider the average criterion. We show that, as discount factor $\alpha$ vanishes, MPI $\nu_{k}^{\alpha, *}\left(i_{k}\right)$ converges to the static index $\nu_{k}^{*}\left(i_{k}\right) \equiv r_{k} \mu_{k}$. This raises the issue of how to break ties when using the latter in a multiclass model, which we resolve by introducing the second-order MPI $\gamma_{k}^{*}\left(i_{k}\right)$, based on the McLaurin expansion $\nu_{k}^{\alpha, *}\left(i_{k}\right)=r_{k} \mu_{k}-\alpha \gamma_{k}^{*}\left(i_{k}\right)+o(\alpha)$ as $\alpha$ vanishes. We obtain $\gamma_{k}^{*}\left(i_{k}\right)$ in closed form. Thus, among classes attaining a tie in first-order MPI $r_{k} \mu_{k}$, higher priority is given to classes with smaller values of second-order MPI $\gamma_{k}^{*}\left(i_{k}\right)$.

For an $\alpha$-discount delay-sensitive class, with $\alpha>0$, we establish PCL-indexability and give a recursion for calculating the discounted MPI $\nu_{k}^{\alpha, *}\left(i_{k}\right)$, where now $i_{k}$ is the number of jobs in system. In what might appear at first sight to be a counterintuitive result, the MPI is nonincreasing in $i_{k}$. Thus, in a multiclass model with such classes scheduled under the MPI policy, ceteris paribus, higher priority is given to shorter queues.

In the delay-sensitive case $c_{k}>0$, in order to obtain an index suitable for the average criterion, we use again a vanishing discount approach. We show that the discounted MPI $\nu_{k}^{\alpha, *}\left(i_{k}\right)$ converges to a limiting index $\nu_{k}^{*}\left(i_{k}\right)$ as $\alpha$ vanishes, and obtain the latter in closed form. We further clarify that such limiting index is indeed an MPI, relative to a new type of indexability introduced in this paper. We term the latter bias indexability, as it emerges from consideration of Blackwell's [1] bias criterion for MDPs.

We interpret the opposite orderings induced on the state space by the MPIs of loss-sensitive and delaysensitive classes in terms of a new structural insight. The fact that the MPI of a loss-sensitive class increases with the queue length means that so does its marginal productivity of work. Namely, reactive work (as the queue gets closer to full) is more productive than preventive work (as it gets closer to empty). In contrast, for a delay-sensitive class preventive work is more productive than reactive work. The intuition behind the latter result is that, when the buffer is full, the delay cost cannot get any worse, and thus larger marginal rates of cost reduction per unit work are achieved as the queue gets shorter.

We obtain relations between the MPI of delay-sensitive classes $k$ and the $c \mu$-rule's index $c_{k} \mu_{k}$. We thus show that, as the buffer size $n_{k}$ grows to infinity, the discounted MPI $\nu_{k}^{\alpha, *}\left(i_{k}\right)$ converges to $c_{k} \mu_{k} / \alpha$. We further show that, in the pure delay-sensitive case, the myopic index defined as the limit of $\alpha \nu_{k}^{\alpha, *}\left(i_{k}\right)$ as $\alpha$ grows to infinity is precisely $c_{k} \mu_{k}$. Finally, we show that, in a multiclass model where classes are delaysensitive, the bias MPI scheduling policy gets closer to the $c \mu$ rule as buffer sizes grow to infinity in fixed proportion to their arrival rates.

We report results of a computational study on the performance of the MPI policies on two-class instances, showing that they are near optimal in every case and often outperform significantly a naive benchmark policy. We further compare the optimal policy's structure with the MPI policy's, finding that the two are often remarkably similar. However, the examples reveal that the structure of optimal policies in the multiclass delay-sensitive case need not be consistent with the state ordering induced by the MPI.

The rest of the paper is organized as follows. Section 2 describes the queueing model and the corresponding scheduling problems of concern. Section 3 reviews our theory of MPIs and PCL-indexability, in a form 
adapted to the model at hand. It further introduces the new concepts of bias MPI and bias PCL-indexability. Section 4 carries out a PCL-indexability analysis for a loss-sensitive class, under discounted and average criteria. Section 5 carries out a corresponding analysis for a delay-sensitive class, under discounted and bias criteria. Finally, Section 6 reports the results of a computational study on the performance of the proposed MPI policies.

\section{Model}

We consider a multiclass $M / M / 1$ queue with a finite dedicated buffer of size $n_{k} \geq 1$ for each class $k \in \mathbb{K} \triangleq$ $\{1, \ldots, K\}$. Class $k$ jobs arrive as a Poisson process with rate $\lambda_{k}$, and their service times are exponentially distributed with rate $\mu_{k}$. Interarrival and service times within and across classes are mutually independent. Upon a job's arrival, if its class' buffer is not full it joins the corresponding queue, and is lost otherwise. We denote by $L_{k}(t)$ the number of class $k$ jobs in system at time $t \geq 0$.

The system controller can choose the nonempty class to be serviced at each job arrival or departure epoch (assume, for concreteness, that jobs within a class are served in FIFO order). Such choices are represented by binary action processes $a_{k}(t)$, where $a_{k}(t)=1$ if the server is working on class $k$ at time $t$, and $a_{k}(t)=0$ otherwise. We thus have the sample-path service-capacity constraint

$$
\sum_{k \in \mathbb{K}} a_{k}(t) \leq 1, \quad t \geq 0
$$

Action choice is dynamically prescribed by a scheduling policy $\pi$. This is chosen from the space $\Pi$ of admissible policies, which are nonanticipative and allow preemptions. Thus, service of a job can be interrupted at any time, and resumed later at the point of interruption.

Regarding the economic structure, the system incurs linear holding and/or rejection costs separably across classes. Class $k$ incurs holding costs at rate $c_{k} \geq 0$ per unit time per job in system; it further incurs rejection costs, at rate $r_{k} \geq 0$ per job lost, with $c_{k}+r_{k}>0$.

It is of interest to consider the following problems: (i) find a discount-optimal scheduling policy,

$$
\min _{\pi \in \Pi} \mathbb{E}^{\pi}\left[\int_{0}^{\infty} e^{-\alpha t} \sum_{k \in \mathbb{K}}\left\{c_{k} L_{k}(t)+r_{k} \lambda_{k} 1_{\left\{L_{k}(t)=n_{k}\right\}}\right\} d t\right],
$$

for a given discount factor $\alpha>0$; and (ii) find an average-optimal scheduling policy,

$$
\min _{\pi \in \Pi} \limsup _{T \rightarrow \infty} \frac{1}{T} \mathbb{E}^{\pi}\left[\int_{0}^{T} \sum_{k \in \mathbb{K}}\left\{c_{k} L_{k}(t)+r_{k} \lambda_{k} 1_{\left\{L_{k}(t)=n_{k}\right\}}\right\} d t\right] .
$$

Given the likely computational intractability of problems (2) and (3), we will not seek to obtain their optimal policies. Instead, our goal will be to design well-grounded and tractable dynamic index policies.

\section{Restless bandits: Indexability, PCL-indexability and the MPI}

We briefly review in this section the key concepts and results of the indexability theory for restless bandits developed in Niño-Mora [7, 8, 10], in a simplified form adapted to the model of concern. We further extend such theory, motivated by phenomena observed in the model's analyses.

Consider a single restless bandit, modeled as a continuous-time MDP whose state $X(t)$ evolves across the finite state space $N \triangleq\left\{m_{0}, \ldots, m_{n}\right\}$. The state ordering $m_{0}, \ldots, m_{n}$ will play a significant role in the sequel. The state space is partitioned into the set $N^{\{0,1\}} \triangleq\left\{m_{1}, \ldots, m_{n}\right\}$ of controllable states, where both the active $(a(t)=1$ : work) and the passive $(a(t)=0$ : rest $)$ actions are available; and the uncontrollable state singleton $N^{\{0\}} \triangleq\left\{m_{0}\right\}$, where only the passive action is available. Holding costs are incurred continuously over time, at the rate of $h^{a}(j) €$ per unit time while the bandit occupies state $j$ and action $a$ prevails. Actions are chosen through adoption of a policy $\pi$, belonging in the class $\Pi$ of admissible policies, which are only required to be nonanticipative. 


\subsection{Discounted MPI}

Consider the case where costs are continuously discounted over time at the exponential rate $\alpha>0$. To evaluate the value of costs incurred under a policy $\pi \in \Pi$, when starting at state $m_{i}$, we use the discounted cost measure

$$
f^{\alpha, \pi}\left(m_{i}\right) \triangleq \mathbb{E}_{m_{i}}^{\pi}\left[\int_{0}^{\infty} e^{-\alpha t} h^{a(t)}(X(t)) d t\right]
$$

where $\mathbb{E}_{m_{i}}^{\pi}[\cdot]$ denotes the corresponding expectation.

We further evaluate the amount of work expended, via the discounted work measure

$$
g^{\alpha, \pi}\left(m_{i}\right) \triangleq \mathbb{E}_{m_{i}}^{\pi}\left[\int_{0}^{\infty} e^{-\alpha t} a(t) d t\right]
$$

To avoid distracting technical issues raised by the choice of initial state, we consider this to be drawn from a distribution assigning a positive probability $p\left(m_{i}\right)>0$ to every state $m_{i}$. We denote the corresponding cost and work measures by $f^{\alpha, \pi}$ and $g^{\alpha, \pi}$, respectively.

Suppose now that work is paid for at the wage rate of $\nu €$ per unit time. We will address the discounted $\nu$-wage problem

$$
\min _{\pi \in \Pi} f^{\alpha, \pi}+\nu g^{\alpha, \pi}
$$

which is to find an admissible policy minimizing the discounted value of holding and working costs.

To solve problem (4), we will postulate (and then establish) that its optimal policies are of threshold type relative to state ordering $m_{0}, m_{1}, \ldots, m_{n}$, so that they prescribe to work in states "above" a threshold state, and to rest otherwise. We represent the policy with threshold state $m_{i}$ by its active-state set

$$
S\left(m_{i}\right) \triangleq \begin{cases}\left\{m_{i+1}, \ldots, m_{n}\right\} & \text { if } 0 \leq i<n \\ \emptyset & \text { if } i=n,\end{cases}
$$

and refer to it as the $S\left(m_{i}\right)$-active policy. The corresponding nested active-state set family is

$$
\mathscr{F} \triangleq\left\{S\left(m_{0}\right), S\left(m_{1}\right), \ldots, S\left(m_{n}\right)\right\} .
$$

We will henceforth refer to such policies as $\mathscr{F}$-policies, writing e.g. $f^{\alpha, S}, g^{\alpha, S}$ for $S \in \mathscr{F}$.

We assume that work measure $g^{\alpha, \pi}$ satisfies the following regularity condition relative to $\mathscr{F}$-policies:

$$
g^{\alpha, S\left(m_{i-1}\right)}>g^{\alpha, S\left(m_{i}\right)}, \quad 1 \leq i \leq n .
$$

i.e. work measure $g^{\alpha, S\left(m_{i}\right)}$ is decreasing in $i$.

We next define a key property based on the structure of optimal policies for problem (4) as the prevailing wage $\nu$ varies.

Definition 3.1 We say that the bandit is $\alpha$-discount $\mathscr{F}$-indexable if there exists an index $\nu^{\alpha, *}: N^{\{0,1\}} \rightarrow \mathbb{R}$, termed the discounted MPI, which is nondecreasing along the state ordering, i.e.

$$
\nu^{\alpha, *}\left(m_{1}\right) \leq \cdots \leq \nu^{\alpha, *}\left(m_{n}\right),
$$

such that, for $0<i<n$, the $S\left(m_{i}\right)$-active policy is optimal for problem (4) iff $\nu \in\left[\nu^{\alpha, *}\left(m_{i}\right), \nu^{\alpha, *}\left(m_{i+1}\right)\right]$.

When it exists, the MPI gives an intuitively appealing rule to solve problem (4): it is optimal to work on the bandit in state $m_{i} \in N^{\{0,1\}}$ iff the latter's MPI value lies at or above the prevailing wage, i.e. iff $\nu^{\alpha, *}\left(m_{i}\right) \geq \nu$. This suggests, drawing on the economic theory of optimal resource allocation, that $\nu^{\alpha, *}\left(m_{i}\right)$ must measure the marginal productivity of work in state $m_{i}$. Such is indeed the case, as established in Niño-Mora [10]. In that paper we further prove the result that the bandit is $\mathscr{F}$-indexable iff it obeys the economics law of diminishing marginal returns (to work), consistently with $\mathscr{F}$-policies. Namely, if one considers the achievable work-cost performance region spanned by points $\left(g^{\alpha, \pi}, f^{\alpha, \pi}\right)$ as $\pi$ ranges over $\Pi$, its lower boundary (efficient frontier) is the piecewise linear and convex function obtained by linear interpolation on points $\left(g^{\alpha, S\left(m_{i}\right)}, f^{\alpha, S\left(m_{i}\right)}\right)$. See Figure 5 for a concrete example in the setting of a delay-sensitive class. The discounted MPI thus has the evaluation

$$
\nu^{\alpha, *}\left(m_{i}\right)=\frac{f^{\alpha, S\left(m_{i}\right)}-f^{\alpha, S\left(m_{i-1}\right)}}{g^{\alpha, S\left(m_{i-1}\right)}-g^{\alpha, S\left(m_{i}\right)}}, \quad 1 \leq i \leq n .
$$




\section{PCL-indexability conditions}

To establish indexability and calculate the MPI, we will deploy the sufficient indexability conditions introduced and developed in Niño-Mora [7, 8, 10], based on satisfaction by performance measures of PCLs. We will not discuss here the PCL framework. For our present purposes, it will suffice to formulate the relevant $P C L$-indexability conditions that need be checked.

We assume that the original continuous-time MDP has been reformulated as a discrete-time MDP via uniformization, so that actions need only be taken at decision epochs given by a Poisson process with a valid uniformization rate $\Lambda$. Given an action $a \in\{0,1\}$ and an active-state set $S \in \mathscr{F}$, denote by $\langle a, S\rangle$ the policy that takes action $a$ in the initial period (between decision epochs), and adopts the $S$-active policy thereafter. For every controllable state $m_{i} \in N^{\{0,1\}}$ and set $S \in \mathscr{F}$, define the discounted $\left(m_{i}, S\right)$-marginal workload by

$$
w^{\alpha, S}\left(m_{i}\right) \triangleq(\alpha+\Lambda)\left(g^{\alpha,\langle 1, S\rangle}\left(m_{i}\right)-g^{\alpha,\langle 0, S\rangle}\left(m_{i}\right)\right),
$$

i.e. $w^{\alpha, S}\left(m_{i}\right)$ measures the marginal rate of increase in work expended which results from working instead of resting in the initial period, provided the $S$-active policy is adopted thereafter.

We analogously define the discounted $\left(m_{i}, S\right)$-marginal cost by

$$
c^{\alpha, S}\left(m_{i}\right) \triangleq(\alpha+\Lambda)\left(f^{\alpha,\langle 0, S\rangle}\left(m_{i}\right)-f^{\alpha,\langle 1, S\rangle}\left(m_{i}\right)\right),
$$

i.e. $c^{\alpha, S}\left(m_{i}\right)$ measures the marginal rate of decrease in cost incurred which results from working instead of resting in the initial period, provided the $S$-active policy is adopted thereafter.

Notice that the inclusion of factor $(\alpha+\Lambda)$ in (8) and (9) has the convenient effect of making $w^{\alpha, S}\left(m_{i}\right)$ and $c^{\alpha, S}\left(m_{i}\right)$ independent of the choice of uniformization rate $\Lambda$.

Define now the discounted $\left(m_{i}, S\right)$-marginal productivity rate, by

$$
\nu^{\alpha, S}\left(m_{i}\right) \triangleq \frac{c^{\alpha, S}\left(m_{i}\right)}{w^{\alpha, S}\left(m_{i}\right)},
$$

provided the denominator does not vanish. Finally, define index $\nu^{\alpha, *}: N^{\{0,1\}} \rightarrow \mathbb{R}$ by

$$
\nu^{\alpha, *}\left(m_{i}\right) \triangleq \nu^{\alpha, S\left(m_{i-1}\right)}\left(m_{i}\right)=\nu^{\alpha, S\left(m_{i}\right)}\left(m_{i}\right), \quad 1 \leq i \leq n,
$$

where the second identity in (11) is proven in Niño-Mora $[8,10]$.

We next use the above to define a tractable class of bandits.

Definition 3.2 We say that the bandit is $\alpha$-discount PCL $(\mathscr{F})$-indexable if the following holds:

(i) Positive marginal workloads: $w^{\alpha, S}\left(m_{i}\right)>0$, for $m_{i} \in N^{\{0,1\}}, S \in \mathscr{F}$.

(ii) Monotone nondecreasing index: $\nu^{\alpha, *}\left(m_{1}\right) \leq \cdots \leq \nu^{\alpha, *}\left(m_{n}\right)$.

Notice that Definition 3.2(i) implies regularity condition (6). See Niño-Mora [8, 10]. We next state the key result used to establish indexability, proven in Niño-Mora $[7,8,10]$ in increasingly general settings.

Theorem 3.3 Discount PCL( $\mathscr{F})$-indexability implies discount $\mathscr{F}$-indexability, with MPI $\nu^{\alpha, *}\left(m_{i}\right)$.

In some models, as will be illustrated in this paper, we have found that marginal workloads $w^{S\left(m_{j}\right)}\left(m_{i}\right)$ are wedge-shaped as $j$ varies, attaining the minimum value at either $j=i-1$ or $j=i$, i.e.

$$
w^{\alpha, S\left(m_{0}\right)}\left(m_{i}\right) \geq \cdots \geq w^{\alpha, S\left(m_{i-1}\right)}\left(m_{i}\right), w^{\alpha, S\left(m_{i}\right)}\left(m_{i}\right) \leq \cdots \leq w^{\alpha, S\left(m_{n}\right)}\left(m_{i}\right), \quad 1 \leq i \leq n .
$$

Such property implies the following insightful characterization of the MPI, proven in Niño-Mora [8, 10].

Theorem 3.4 Suppose that the bandit is $\alpha$-discount PCL(F)-indexable and condition (12) holds. Then

$$
\max _{m_{i} \in S \in \mathscr{F}} \nu^{\alpha, S}\left(m_{i}\right)=\nu^{\alpha, *}\left(m_{i}\right)=\min _{m_{i} \notin S \in \mathscr{F}} \nu^{\alpha, S}\left(m_{i}\right), \quad m_{i} \in N^{\{0,1\}} .
$$

Theorem 3.4 characterizes the MPI as an optimal marginal productivity rate relative to $\mathscr{F}$-policies, in a dual max-min relation. 


\subsection{Average and second-order MPI}

The MPI can be defined relative to generic cost and work measures $f^{\pi}$ and $g^{\pi}$, as discussed in Niño-Mora [10]. Thus, under the average criterion we take

$$
f^{\pi} \triangleq \limsup _{T \rightarrow \infty} \frac{1}{T} \mathbb{E}^{\pi}\left[\int_{0}^{T} h^{a(t)}(X(t)) d t\right] \quad \text { and } \quad g^{\pi} \triangleq \limsup _{T \rightarrow \infty} \frac{1}{T} \mathbb{E}^{\pi}\left[\int_{0}^{T} a(t) d t\right],
$$

where as above we assume the initial state to be drawn from an arbitrary positive probability mass function. We can thus readily extend Definition 3.1 to define the concepts of average $\mathscr{F}$-indexability and average MPI $\nu^{*}\left(m_{i}\right)$, based on the structure of optimal policies for the average $\nu$-wage problem

$$
\min _{\pi \in \Pi} f^{\pi}+\nu g^{\pi}
$$

Similarly as for the discounted case, we assume that average work measure $g^{\pi}$ satisfies the following regularity condition relative to $\mathscr{F}$-policies:

$$
g^{S\left(m_{i-1}\right)}>g^{S\left(m_{i}\right)}, \quad 1 \leq i \leq n .
$$

i.e. work measure $g^{S\left(m_{i}\right)}$ is decreasing in $i$.

Definition 3.5 We say that the bandit is average $\mathscr{F}$-indexable if there exists an index $\nu^{*}: N^{\{0,1\}} \rightarrow \mathbb{R}$, termed the average MPI, which is nondecreasing along the state ordering, i.e.

$$
\nu^{*}\left(m_{1}\right) \leq \cdots \leq \nu^{*}\left(m_{n}\right)
$$

such that, for $0<i<n$, the $S\left(m_{i}\right)$-active policy is optimal for problem (13) iff $\nu \in\left[\nu^{*}\left(m_{i}\right), \nu^{*}\left(m_{i+1}\right)\right]$.

The above PCL-indexability conditions are readily extended to the average criterion. Under the latter, we use the average $\left(m_{i}, S\right)$-marginal workload, average $\left(m_{i}, S\right)$-marginal cost and average $\left(m_{i}, S\right)$-marginal productivity rate, defined for $m_{i} \in N^{\{0,1\}}$ and $S \in \mathscr{F}$ by

$$
\begin{gathered}
w^{S}\left(m_{i}\right) \triangleq \lim _{T \rightarrow \infty} \mathbb{E}_{m_{i}}^{\langle 1, S\rangle}\left[\int_{0}^{T} a(t) d t\right]-\mathbb{E}_{m_{i}}^{\langle 0, S\rangle}\left[\int_{0}^{T} a(t) d t\right]=\lim _{\alpha \searrow 0} w^{\alpha, S}\left(m_{i}\right), \\
c^{S}\left(m_{i}\right) \triangleq \lim _{T \rightarrow \infty} \mathbb{E}_{m_{i}}^{\langle 0, S\rangle}\left[\int_{0}^{T} h^{a(t)}(X(t)) d t\right]-\mathbb{E}_{m_{i}}^{\langle 1, S\rangle}\left[\int_{0}^{T} h^{a(t)}(X(t)) d t\right]=\lim _{\alpha \searrow 0} c^{\alpha, S}\left(m_{i}\right),
\end{gathered}
$$

and

$$
\nu^{S}\left(m_{i}\right) \triangleq \frac{c^{S}\left(m_{i}\right)}{w^{S}\left(m_{i}\right)}=\lim _{\alpha \searrow 0} \nu^{\alpha, S}\left(m_{i}\right)
$$

respectively. We further define index $\nu^{*}: N^{\{0,1\}} \rightarrow \mathbb{R}$ by

$$
\nu^{*}\left(m_{i}\right) \triangleq \nu^{S\left(m_{i-1}\right)}\left(m_{i}\right)=\nu^{S\left(m_{i}\right)}\left(m_{i}\right)=\lim _{\alpha \searrow 0} \nu^{\alpha, *}\left(m_{i}\right), \quad m_{i} \in N^{\{0,1\}} .
$$

Notice that we have indicated the limiting relations between corresponding undiscounted and discounted terms as the discount factor vanishes.

We can thus readily extend Definition 3.2 to define the concept of average PCL( $\mathscr{F})$-indexable bandits, and obtain the average-criterion counterparts of Theorems 3.3 and 3.4.

Definition 3.6 We say that the bandit is average $\operatorname{PCL}(\mathscr{F})$-indexable if the following holds:

(i) Positive marginal workloads: $w^{S}\left(m_{i}\right)>0$, for $m_{i} \in N^{\{0,1\}}, S \in \mathscr{F}$.

(ii) Monotone nondecreasing index: $\nu^{*}\left(m_{1}\right) \leq \cdots \leq \nu^{*}\left(m_{n}\right)$.

Theorem 3.7 Average PCL( $\mathscr{F})$-indexability implies average $\mathscr{F}$-indexability, with MPI $\nu^{*}\left(m_{i}\right)$. 
In some models, as will be illustrated in this paper, the average MPI is constant across states, i.e. $\nu^{*}\left(m_{i}\right) \equiv$ $\nu^{*}$ for $m_{i} \in N^{\{0,1\}}$. This raises the issue of how to discriminate between states, i.e. how to break ties, when using an MPI-based scheduling policy in a multi-project setting, where the MPI of several bandits coincides. We propose to resolve such issue by considering the second-order McLaurin expansion of the discounted MPI as the discount factor vanishes,

$$
\nu^{\alpha, *}\left(m_{i}\right)=\nu^{*}-\gamma^{*}\left(m_{i}\right) \alpha+o(\alpha), \quad \text { as } \alpha \searrow 0
$$

and then defining the second-order MPI by

$$
\gamma^{*}\left(m_{i}\right) \triangleq \lim _{\alpha \searrow 0} \frac{\nu^{*}-\nu^{\alpha, *}\left(m_{i}\right)}{\alpha}, \quad m_{i} \in N^{\{0,1\}} .
$$

Notice that the second-order MPI is monotone nonincreasing along the state ordering, i.e.

$$
\gamma^{*}\left(m_{1}\right) \geq \gamma^{*}\left(m_{2}\right) \geq \cdots \geq \gamma^{*}\left(m_{n}\right) .
$$

\subsection{Bias MPI}

It is well known in MDP theory that the average-optimality criterion can be underselective, in that there may be multiple average-optimal policies. In the present setting, this can lead to nonexistence of the average MPI.

To deal with such phenomena, we introduce next the concepts of bias $\mathscr{F}$-indexability and bias MPI, based on Blackwell's [1] more sensitive bias-optimality criterion. See Lewis and Puterman [5] for a survey of work on the bias criterion in MDPs. Previously, we had introduced and deployed in Niño-Mora [10] the concepts of average-bias $\mathscr{F}$-indexability and average-bias MPI, based on mixing an average cost measure with a bias work measure.

We assume that the bandit is $\alpha$-discount $\mathscr{F}$-indexable for all $\alpha$ close enough to 0 .

Definition 3.8 We say that the bandit is bias $\mathscr{F}$-indexable if there exists an index $\nu^{*}: N^{\{0,1\}} \rightarrow \mathbb{R}$, termed the bias MPI, which is nondecreasing along the state ordering, i.e.

$$
\nu^{*}\left(m_{1}\right) \leq \cdots \leq \nu^{*}\left(m_{n}\right)
$$

such that, for $0<i<n$, the $S\left(m_{i}\right)$-active policy is bias optimal for (13) iff $\nu \in\left[\nu^{*}\left(m_{i}\right), \nu^{*}\left(m_{i+1}\right)\right]$.

We will find it convenient to use the following definition of bias optimality, drawing on its relation with 0 -discount optimality as established in Puterman [11, Theorem 10.1.6]: we say that a stationary policy $S$ is bias optimal for $\nu$-wage problem (13) if, for any average-optimal stationary policy $\pi$, it holds that

$$
\liminf _{\alpha \searrow 0} v^{\alpha, \pi}(\nu)-v^{\alpha, S}(\nu) \geq 0 .
$$

\section{PCL-indexability analysis: loss-sensitive classes}

We address in this section the PCL-indexability analysis for the restless bandit model corresponding to a loss-sensitive class in isolation, i.e. an $M / M / 1 / n$ queue with arrival and service rates $\lambda$ and $\mu$, respectively, subject to service control, with delay cost rate $c \geq 0$ and rejection cost rate $r>0$ satisfying $\alpha r \geq c$.

For such a class, we will find it convenient to define the state by $X(t) \triangleq n-L(t)$, the number of empty buffer spaces. We will use the state ordering $m_{i} \triangleq n-i$ for $0 \leq i \leq n$, so that $N \triangleq\{n, n-1, \ldots, 0\}$, $N^{\{0,1\}} \triangleq\{n-1, \ldots, 0\}, N^{\{0\}} \triangleq\{n\}$, and the active-state sets in $\mathscr{F}$ are given by $S(0) \triangleq \emptyset$, and

$$
S(i) \triangleq\{i-1, \ldots, 0\}, \quad 1 \leq i \leq n .
$$

In words, $\mathscr{F}$-policies prescribe the server to work when the number of empty buffer spaces is small enough.

\subsection{Discounted criterion}

We start by laying the groundwork for calculations of discounted marginal workloads and costs. We use uniformization to obtain an equivalent discrete-time MDP, where the state is sampled at epochs of a Poisson process with rate $\Lambda \geq \lambda+\mu$, which include arrival and service completion instants, along with dummy 
transition epochs. The uniformized MDP's state transition probability matrix $\mathbf{P}^{a}=\left(p_{i j}^{a}\right)$ under action $a \in$ $\{0,1\}$ is given by

$$
p_{i j}^{a} \triangleq \begin{cases}\lambda / \Lambda & \text { if } 0 \leq j=i-1 \leq n-1 \\ (\Lambda-\lambda-\mu a) / \Lambda & \text { if } 0<j=i<n \\ (\Lambda-\mu a) / \Lambda & \text { if } j=i=0 \\ (\Lambda-\lambda) / \Lambda & \text { if } j=i=n \\ \mu a / \Lambda & \text { if } 1 \leq j=i+1 \leq n \\ 0 & \text { otherwise. }\end{cases}
$$

Notice that we take both actions to have the same effect on uncontrollable state $n$.

The corresponding discrete-time discount factor is $\Lambda /(\alpha+\Lambda)$, and the discrete-time one-period cost rate in state $i$ is $\left\{c(n-i)+r \lambda 1_{\{i=0\}}\right\} /(\alpha+\Lambda)$.

Consider now discounted marginal workloads $w^{\alpha, S}(i)$, for $i \in N^{\{0,1\}}$ and $S \in \mathscr{F}$. From their definition in (8) and uniformization we readily obtain

$$
w^{\alpha, S}(i)=1+\mu \Delta g^{\alpha, S}(i+1), \quad 0 \leq i \leq n-1,
$$

where $\Delta g^{\alpha, S}(i) \triangleq g^{\alpha, S}(i)-g^{\alpha, S}(i-1)$. Hence, calculation of the $w^{\alpha, S}(i)$ 's reduces to that of the $\Delta g^{\alpha, S}(i)$ 's. We thus start by characterizing work measures $g^{\alpha, S}(i)$. We denote below by $\mu_{S}(i)$ the effective service rate in state $i$ under the $S$-active policy, i.e. letting $1_{S}(i)$ be the indicator function of $S$,

$$
\mu_{S}(i) \triangleq \mu 1_{S}(i), \quad 0 \leq i \leq n .
$$

The next result gives the standard evaluation equations for the $g^{\alpha, S}(i)$ 's, for fixed $S \in \mathscr{F}$.

Lemma 4.1 Discounted work measures $g^{\alpha, S}(i)$, for $i \in N$, are characterized by the equations

$$
\begin{aligned}
\alpha g^{\alpha, S}(0) & =1_{S}(0)+\mu_{S}(0) \Delta g^{\alpha, S}(1) \\
\alpha g^{\alpha, S}(i) & =1_{S}(i)-\lambda \Delta g^{\alpha, S}(i)+\mu_{S}(i) \Delta g^{\alpha, S}(i+1), \quad 1 \leq i \leq n-1 \\
\alpha g^{\alpha, S}(n) & =-\lambda \Delta g^{\alpha, S}(n) .
\end{aligned}
$$

The next result, characterizing first-order differences $\Delta g^{\alpha, S}(i)$, follows immediately.

Lemma 4.2 Terms $\Delta g^{S}(i)$, for $1 \leq i \leq n$, are characterized by the equations

$$
\begin{aligned}
\left(\alpha+\lambda+\mu_{S}(0)\right) \Delta g^{\alpha, S}(1) & =\Delta 1_{S}(1)+\mu_{S}(1) \Delta g^{\alpha, S}(2) \\
\left(\alpha+\lambda+\mu_{S}(i-1)\right) \Delta g^{\alpha, S}(i) & =\Delta 1_{S}(i)+\lambda \Delta g^{\alpha, S}(i-1)+\mu_{S}(i) \Delta g^{\alpha, S}(i+1), \quad 2 \leq i \leq n-1 \\
\left(\alpha+\lambda+\mu_{S}(n-1)\right) \Delta g^{\alpha, S}(n) & =-1_{S}(n-1)+\lambda \Delta g^{\alpha, S}(n-1) .
\end{aligned}
$$

We can now give the evaluation equations for discounted marginal workloads.

Lemma 4.3 Marginal workloads $w^{\alpha, S}(i)$, for $i \in N^{\{0,1\}}$, are characterized by the equations

$$
\begin{aligned}
\left(\alpha+\lambda+\mu_{S}(0)\right) w^{\alpha, S}(0) & =\alpha+\lambda+\mu_{S}(1) w^{\alpha, S}(1) \\
\left(\alpha+\lambda+\mu_{S}(i)\right) w^{\alpha, S}(i) & =\alpha+\lambda w^{\alpha, S}(i-1)+\mu_{S}(i+1) w^{\alpha, S}(i+1), \quad 1 \leq i \leq n-2 \\
\left(\alpha+\lambda+\mu_{S}(n-1)\right) w^{\alpha, S}(n-1) & =\alpha+\lambda w^{\alpha, S}(n-2) .
\end{aligned}
$$

Proof. The result follows immediately from identity (20) and Lemma 4.2.

We next turn attention to discounted marginal costs $c^{\alpha, S}(i)$, for $i \in N^{\{0,1\}}$ and $S \in \mathscr{F}$. From their definition in (9) and uniformization we readily obtain

$$
c^{\alpha, S}(i)=-\mu \Delta f^{\alpha, S}(i+1) .
$$

Hence, we need to characterize first-order differences $\Delta f^{\alpha, S}(i)$.

Proceeding as before, we next give the standard evaluation equations for the $f^{\alpha, S}(i)$ 's. 
Lemma 4.4 Discounted cost measures $f^{\alpha, S}(i)$, for $i \in N$, are characterized by the equations

$$
\begin{aligned}
\alpha f^{\alpha, S}(0) & =c n+r \lambda+\mu_{S}(0) \Delta f^{\alpha, S}(1) \\
\alpha f^{\alpha, S}(i) & =c(n-i)-\lambda \Delta f^{\alpha, S}(i)+\mu_{S}(i) \Delta f^{\alpha, S}(i+1), 1 \leq i \leq n-1 \\
\alpha f^{\alpha, S}(n) & =-\lambda \Delta f^{\alpha, S}(n) .
\end{aligned}
$$

The next result, characterizing first-order differences $\Delta f^{\alpha, S}(i)$, follows immediately.

Lemma 4.5 Terms $\Delta f^{\alpha, S}(i)$, for $1 \leq i \leq n$, are characterized by the equations

$$
\begin{aligned}
\left(\alpha+\lambda+\mu_{S}(0)\right) \Delta f^{\alpha, S}(1) & =-(c+r \lambda)+\mu_{S}(1) \Delta f^{\alpha, S}(2) \\
\left(\alpha+\lambda+\mu_{S}(i-1)\right) \Delta f^{\alpha, S}(i) & =-c+\lambda \Delta f^{\alpha, S}(i-1)+\mu_{S}(i) \Delta f^{\alpha, S}(i+1), 2 \leq i \leq n-1 \\
\left(\alpha+\lambda+\mu_{S}(n-1)\right) \Delta f^{\alpha, S}(n) & =-c+\lambda \Delta f^{\alpha, S}(n-1) .
\end{aligned}
$$

We can now give the evaluation equations for discounted marginal costs.

Lemma 4.6 Marginal costs $c^{\alpha, S}(i)$, for $i \in N^{\{0,1\}}$, are characterized by the equations

$$
\begin{aligned}
\left(\alpha+\lambda+\mu_{S}(0)\right) c^{\alpha, S}(0) & =(c+r \lambda) \mu+\mu_{S}(1) c^{\alpha, S}(1) \\
\left(\alpha+\lambda+\mu_{S}(i)\right) c^{\alpha, S}(i) & =c \mu+\lambda c^{\alpha, S}(i-1)+\mu_{S}(i+1) c^{\alpha, S}(i+1), 1 \leq i \leq n-2 \\
\left(\alpha+\lambda+\mu_{S}(n-1)\right) c^{\alpha, S}(n-1) & =c \mu+\lambda c^{\alpha, S}(n-2) .
\end{aligned}
$$

Proof. The result follows immediately from identity (21) and Lemma 4.2.

\subsubsection{Discounted marginal workloads: calculation and properties}

We next draw on the above to calculate discounted marginal workloads, and to establish their required properties.

We will develop a recursion to solve the system of evaluation equations in Lemma 4.3 for every activestate set $S(j)$. Notice that the case $j=0$ is trivial, since $S(0)=\emptyset$, and hence $w^{\alpha, S(0)}(i) \equiv 1$. For other cases, calculations will proceed by upward recursion on $j$. We start by solving the system for $j=1$, whence the first equation gives pivot term

$$
w^{\alpha, S(1)}(0)=\frac{\alpha+\lambda}{\alpha+\lambda+\mu} .
$$

From the remaining equations, we calculate recursively $w^{\alpha, S(1)}(i)$, for $1 \leq i \leq n-1$.

Similarly, if for a given $1 \leq j \leq n$ pivot term $w^{\alpha, S(j)}(j-1)$ were available, from the remaining equations for $S(j)$ we could recursively calculate remaining terms $w^{\alpha, S(j)}(i)$. Therefore, if we could represent pivot $w^{\alpha, S(j+1)}(j)$ in terms of previous pivot $w^{\alpha, S(j)}(j-1)$, for every $1 \leq j \leq n-1$, such relations would furnish the backbone of a recursion to calculate all marginal workloads $w^{\alpha, S(j)}(i)$.

We next set out to relate successive pivots. We will use the following vectors (where $\mathrm{x}^{\mathrm{T}}$ denotes the transpose of a vector $\mathbf{x}, \mathbf{1}$ denotes a vector of ones, and $\mathbf{e}_{k}$ denotes the $k$ th unit coordinate vector of the appropriate dimension): for $1 \leq j \leq n$, let

$$
\mathbf{w}^{j} \triangleq\left[w^{\alpha, S(j)}(0) \quad \cdots \quad w^{\alpha, S(j)}(j-1)\right]^{\mathrm{T}}, \quad \mathbf{b}^{j} \triangleq \frac{\alpha}{\alpha+\lambda+\mu} \mathbf{1}+\frac{\lambda}{\alpha+\lambda+\mu} \mathbf{e}_{1} ;
$$

and, for $1 \leq j \leq n-1$, let

$$
\widehat{\mathbf{w}}^{j} \triangleq\left[w^{\alpha, S(j+1)}(0) \quad \cdots \quad w^{\alpha, S(j+1)}(j-1)\right]^{\mathrm{T}}, \quad \widehat{\mathbf{b}}^{j} \triangleq \mathbf{b}^{j}+\frac{\mu w^{\alpha, S(j+1)}(j)}{\alpha+\lambda+\mu} \mathbf{e}_{j} .
$$

Let us further introduce, for $1 \leq j \leq n$, the square matrix of dimension $j$

$$
\mathbf{B}^{j} \triangleq \frac{1}{\alpha+\lambda+\mu}\left[\begin{array}{ccccc}
0 & \mu & & & \\
\lambda & 0 & \mu & & \\
& \ddots & \ddots & \ddots & \\
& & \lambda & 0 & \mu \\
& & & \lambda & 0
\end{array}\right],
$$

with $\mathbf{B}^{1} \triangleq 0$. The next result reformulates some equations in Lemma 4.3. 


\section{Lemma 4.7}

(a) $\mathbf{w}^{j}=\mathbf{b}^{j}+\mathbf{B}^{j} \mathbf{w}^{j}, \quad 1 \leq j \leq n$.

(b) $\widehat{\mathbf{w}}^{j}=\widehat{\mathbf{b}}^{j}+\mathbf{B}^{j} \widehat{\mathbf{w}}^{j}, \quad 1 \leq j \leq n-1$.

To proceed, we introduce coefficients $q^{\alpha}(0), \ldots, q^{\alpha}(n-1)$, defined by

$$
q^{\alpha}(j) \triangleq \begin{cases}1 & \text { if } j=0 \\ \frac{\operatorname{det}\left(\mathbf{I}-\mathbf{B}^{j+1}\right)}{\operatorname{det}\left(\mathbf{I}-\mathbf{B}^{j}\right)} & \text { if } 1 \leq j \leq n-1,\end{cases}
$$

where I denotes the identity matrix of the appropriate dimension. The next result shows that such coefficients are well defined, and establishes properties on which we will draw in the ensuing analyses.

Lemma 4.8 Terms $q^{\alpha}(j)$ are well defined, and satisfy the following properties:

(a) $q^{\alpha}(j)>0, \quad 0 \leq j \leq n-1$.

(b) They can be computed by upward recursion, setting $q^{\alpha}(0)=1$ and

$$
q^{\alpha}(j)=1-\frac{\lambda \mu}{(\alpha+\lambda+\mu)^{2} q^{\alpha}(j-1)}, \quad 1 \leq j \leq n-1 .
$$

(c) $\frac{\alpha+\lambda}{\alpha+\lambda+\mu}<q^{\alpha}(j)<1, \quad 1 \leq j \leq n-1$.

Proof. (a) The row sums of matrix $\mathbf{B}^{j}$ are less than unity, and hence so is its spectral radius. Therefore, $\operatorname{det}\left(\mathbf{I}-\mathbf{B}^{j}\right)>0$ and the $q^{\alpha}(j)$ 's are well defined and positive.

(b) The recursion follows from definition of $q^{\alpha}(j)$ and the linear algebra identities

$$
\begin{aligned}
\operatorname{det}\left(\mathbf{I}-\mathbf{B}^{2}\right) & =\operatorname{det}\left(\mathbf{I}-\mathbf{B}^{1}\right)-\frac{\lambda \mu}{(\alpha+\lambda+\mu)^{2}} \\
\operatorname{det}\left(\mathbf{I}-\mathbf{B}^{j+1}\right) & =\operatorname{det}\left(\mathbf{I}-\mathbf{B}^{j}\right)-\frac{\lambda \mu}{(\alpha+\lambda+\mu)^{2}} \operatorname{det}\left(\mathbf{I}-\mathbf{B}^{j-1}\right), \quad 2 \leq j \leq n-1 .
\end{aligned}
$$

(c) Parts (a) and (b) give that $q^{\alpha}(j)<1$ for $1 \leq j \leq n-1$. We next argue that

$$
q^{\alpha}(j)>\frac{\alpha+\lambda}{\alpha+\lambda+\mu}, \quad 0 \leq j \leq n-1,
$$

by upward induction on $j$. The case $j=0$ is trivial. Suppose that the result holds for some $0 \leq j \leq n-2$. Then, part (b) and the induction hypothesis yield

$$
q^{\alpha}(j+1)=1-\frac{\mu}{\alpha+\lambda+\mu} \frac{\frac{\lambda}{\alpha+\lambda+\mu}}{q^{\alpha}(j)}>1-\frac{\mu}{\alpha+\lambda+\mu}=\frac{\alpha+\lambda}{\alpha+\lambda+\mu} .
$$

Therefore, (24) holds for $0 \leq j \leq n-1$. This completes the proof.

We are now ready to relate successive pivots.

\section{Lemma 4.9}

$$
q^{\alpha}(j) w^{\alpha, S(j+1)}(j)=\frac{\alpha+\lambda w^{\alpha, S(j)}(j-1)}{\alpha+\lambda+\mu}, \quad 1 \leq j \leq n-1 .
$$


Calculation of $w^{\alpha, S(0)}(i)$ 's (note: $S(0)=\emptyset$ ):

$$
w^{\alpha, S(1)}(i)=1, \quad 0 \leq i \leq n-1
$$

Calculation of $w^{\alpha, S(1)}(i)$ 's:

$$
w^{\alpha, S(1)}(0)=\frac{\alpha+\lambda}{\alpha+\lambda+\mu} ; \quad w^{\alpha, S(1)}(i)=\frac{\alpha+\lambda w^{\alpha, S(1)}(i-1)}{\alpha+\lambda}, \quad 2 \leq i \leq n-1
$$

Calculation of $w^{\alpha, S(j)}(i)$ 's, for $2 \leq j \leq n$ :

$$
\begin{aligned}
w^{\alpha, S(j)}(j-1) & =\frac{\alpha+\lambda w^{\alpha, S(j-1)}(j-2)}{(\alpha+\lambda+\mu) q^{\alpha}(j-1)} \\
w^{\alpha, S(j)}(j-2) & =\frac{-\alpha+(\alpha+\lambda+\mu) w^{\alpha, S(j)}(j-1)}{\lambda} \\
w^{\alpha, S(j)}(i) & =\frac{\alpha+\lambda w^{\alpha, S(j)}(i-1)}{\alpha+\lambda}, \quad j \leq i \leq n-1 \\
w^{\alpha, S(j)}(i) & =\frac{-\alpha-\mu w^{\alpha, S(j)}(i+2)+(\alpha+\lambda+\mu) w^{\alpha, S(j)}(i+1)}{\lambda}, 1 \leq i \leq j-3 \\
w^{\alpha, S(j)}(0) & =\frac{\alpha+\lambda+\mu w^{\alpha, S(j)}(1)}{\alpha+\lambda+\mu}
\end{aligned}
$$

Figure 1: Marginal workloads: recursive calculation.

Proof. Fix $1 \leq j \leq n-1$. By Lemma 4.7 and the definitions of $\mathbf{b}^{j}, \widehat{\mathbf{b}}^{j}$, we have

$$
\widehat{\mathbf{w}}^{j}-\mathbf{w}^{j}=\left(\mathbf{I}-\mathbf{B}^{j}\right)^{-1}\left(\widehat{\mathbf{b}}^{j}-\mathbf{b}^{j}\right)=\frac{\mu w^{\alpha, S(j+1)}(j)}{\alpha+\lambda+\mu}\left(\mathbf{I}-\mathbf{B}^{j}\right)^{-1} \mathbf{e}_{j} .
$$

Now, noting that the element in position $(j, j)$ of matrix $\left(\mathbf{I}-\mathbf{B}^{j}\right)^{-1}$ has the evaluation 1 if $j=1$, and $\operatorname{det}\left(\mathbf{I}-\mathbf{B}^{j-1}\right) / \operatorname{det}\left(\mathbf{I}-\mathbf{B}^{j}\right)$ if $j \geq 2$, which in either case equals $1 / q^{\alpha}(j-1)$, it follows from (25) that

$$
w^{\alpha, S(j+1)}(j-1)-w^{\alpha, S(j)}(j-1)=\frac{\mu}{q^{\alpha}(j-1)} \frac{w^{\alpha, S(j+1)}(j)}{\alpha+\lambda+\mu} .
$$

We next substitute for $w^{\alpha, S(j+1)}(j-1)$ in (26) using Lemma 4.3's identity

$$
w^{\alpha, S(j+1)}(j)=\frac{\alpha}{\alpha+\lambda+\mu}+\frac{\lambda}{\alpha+\lambda+\mu} w^{\alpha, S(j+1)}(j-1),
$$

and further substitute for $q^{\alpha}(j-1)$ in terms of $q^{\alpha}(j)$ using Lemma 4.8(b), to obtain, after straightforward algebra, the stated identity.

We can now give a complete recursion for calculating discounted marginal workloads, as shown in Figure 1. Figure 2 further clarifies the recursion, showing by arrows the directions in which calculations proceed, and enclosing in boxes the pivot terms, which furnish the recursion's backbone.

Proposition 4.10 Discounted marginal workloads $w^{\alpha, S}(i)$, for $i \in N^{\{0,1\}}$ and $S \in \mathscr{F}$, are calculated by the recursion shown in Figure 1.

Proof. The result follows directly from Lemmas 4.3 and 4.9.

We next use the above to establish required properties of discounted marginal workloads. Figure 2 illustrates the inequalities presented in the following result.

Lemma 4.11 Discounted marginal workloads satisfy the following inequalities:

(a) $w^{\alpha, S(j+1)}(j)>0, \quad 0 \leq j \leq n-1$. 


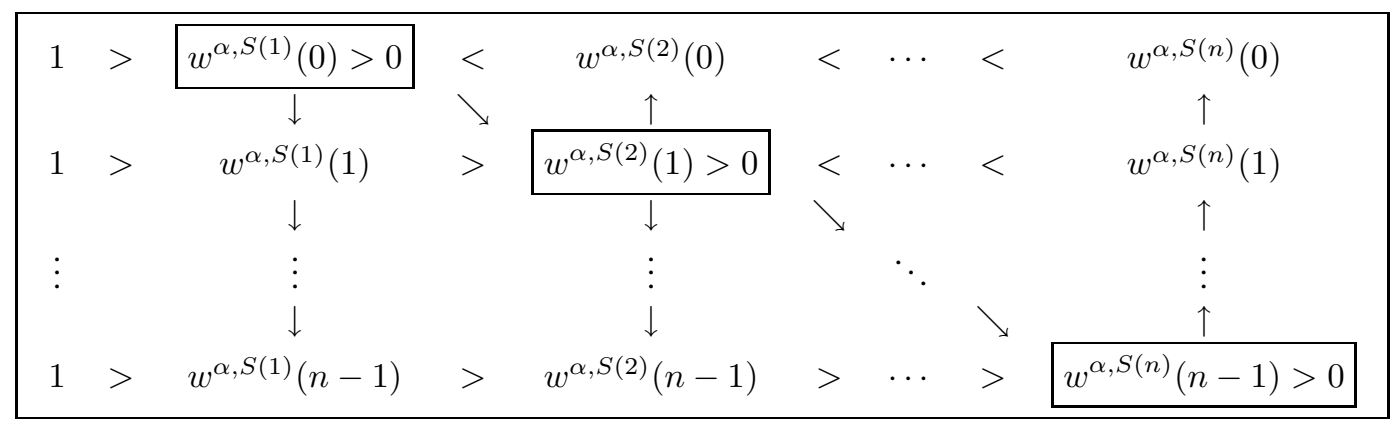

Figure 2: Marginal workloads: directions of calculations and wedge-shape property.

(b) $w^{\alpha, S(j+1)}(i)>w^{\alpha, S(j)}(i), \quad 0 \leq i \leq j-1, j \leq n-1$.

(c) $w^{\alpha, S(j)}(i)>w^{\alpha, S(j+1)}(i), \quad 0 \leq j \leq i \leq n-1$.

Proof. (a) This part follows by upward induction on $j$, using (22) and Lemma 4.9.

(b) Take $1 \leq j \leq n-1$. Since the spectral radius of matrix $\mathbf{B}^{j}$ is less than unity (cf. Lemma 4.8's proof), it follows that matrix $\left(\mathbf{I}-\mathbf{B}^{j}\right)^{-1}$ is positive componentwise, which in turn implies $\left(\mathbf{I}-\mathbf{B}^{j}\right)^{-1} \mathbf{e}_{j}>\mathbf{0}$. Combining such result with part (a) and identity (25), we obtain $\widehat{\mathbf{w}}^{j}-\mathbf{w}^{j}>\mathbf{0}$, i.e.

$$
w^{\alpha, S(j+1)}(i)>w^{\alpha, S(j)}(i), \quad 0 \leq i \leq j-1,
$$

as required.

(c) Let $0 \leq j \leq n-2$. By Lemma 4.3, we have

$$
\begin{aligned}
(\alpha+\lambda) w^{\alpha, S(j)}(i) & =\alpha+\lambda w^{\alpha, S(j)}(i-1), \quad j \leq i \leq n-1 \\
(\alpha+\lambda) w^{\alpha, S(j+1)}(i) & =\alpha+\lambda w^{\alpha, S(j+1)}(i-1), \quad j+1 \leq i \leq n-1,
\end{aligned}
$$

whence we obtain

$$
w^{\alpha, S(j)}(i)-w^{\alpha, S(j+1)}(i)=\frac{\lambda}{\alpha+\lambda}\left\{w^{\alpha, S(j)}(i-1)-w^{\alpha, S(j+1)}(i-1)\right\}, \quad j+1 \leq i \leq n-1 .
$$

In light of the latter identities, to prove the required result it suffices to establish that

$$
w^{\alpha, S(j)}(j)-w^{\alpha, S(j+1)}(j)>0, \quad 0 \leq j \leq n-1,
$$

which we set out to do next.

The case $j=0$ follows from

$$
w^{\alpha, S(0)}(0)-w^{\alpha, S(1)}(0)=1-\frac{\alpha+\lambda}{\alpha+\lambda+\mu}=\frac{\mu}{\alpha+\lambda+\mu}>0 .
$$

For $1 \leq j \leq n-1$, drawing again on Lemma 4.3, we can write

$$
\begin{aligned}
(\alpha+\lambda+\mu) w^{\alpha, S(j+1)}(j) & =\alpha+\lambda w^{\alpha, S(j+1)}(j-1) \\
(\alpha+\lambda) w^{\alpha, S(j)}(j) & =\alpha+\lambda w^{\alpha, S(j)}(j-1) .
\end{aligned}
$$

Using in turn the last two identities, (26), part (a) and Lemma 4.8(c), yields

$$
\begin{aligned}
(\alpha+\lambda)\left\{w^{\alpha, S(j)}(j)-w^{\alpha, S(j+1)}(j)\right\} & =\mu w^{\alpha, S(j+1)}(j)-\lambda\left\{w^{\alpha, S(j+1)}(j-1)-w^{\alpha, S(j)}(j-1)\right\} \\
& =\left\{1-\frac{\lambda / q^{\alpha}(j-1)}{\alpha+\lambda+\mu}\right\} \mu w^{\alpha, S(j+1)}(j)>0,
\end{aligned}
$$

as required. This completes the proof.

We can now give the key properties of discounted marginal workloads. 


\section{Proposition 4.12 (Positive and wedge-shaped discounted marginal workloads)}

(a) $w^{\alpha, S}(i)>0$, for $i \in N^{\{0,1\}}$ and $S \in \mathscr{F}$.

(b) The $w^{\alpha, S(j)}(i)$ 's are wedge-shaped as $j$ varies, as shown in Figure 2, so that condition (12) holds with strict inequalities.

Proof. Both parts follow immediately from Lemma 4.11.

\subsubsection{Discounted marginal costs: calculation}

We next set out to calculate the required discounted marginal costs $c^{\alpha, S}(i)$, proceeding similarly as before for discounted marginal workloads. Only pivot terms $c^{\alpha, S(j+1)}(j)$, for $0 \leq j \leq n-1$, are required for the PCL-indexability analysis. We next develop a recursion to calculate them, along the lines followed above to calculate the $w^{\alpha, S(j+1)}(j)$ 's.

We start by noting that Lemma 4.6 readily yields the first such pivot as

$$
c^{\alpha, S(1)}(0)=\frac{c+r \lambda}{\alpha+\lambda+\mu} \mu .
$$

We next set out to relate successive pivots. Define the following vectors: for $1 \leq j \leq n$, let

$$
\mathbf{c}^{j}=\left[\begin{array}{lll}
c^{\alpha, S(j)}(0) & \cdots & c^{\alpha, S(j)}(j-1)
\end{array}\right]^{\mathrm{T}}, \quad \mathbf{h}^{j}=\frac{c \mu}{\alpha+\lambda+\mu} \mathbf{1}+\frac{r \lambda \mu}{\alpha+\lambda+\mu} \mathbf{e}_{1} ;
$$

and, for $1 \leq j \leq n-1$, let

$$
\widehat{\mathbf{c}}^{j}=\left[\begin{array}{lll}
c^{\alpha, S(j+1)}(0) & \cdots & c^{\alpha, S(j+1)}(j-1)
\end{array}\right]^{\mathrm{T}}, \quad \widehat{\mathbf{h}}^{j}=\mathbf{h}^{j}+\frac{\mu c^{\alpha, S(j+1)}(j)}{\alpha+\lambda+\mu} \mathbf{e}_{j} .
$$

The following result is a counterpart of Lemma 4.7.

\section{Lemma 4.13}

(a) $\mathbf{c}^{j}=\mathbf{h}^{j}+\mathbf{B}^{j} \mathbf{c}^{j}, \quad 1 \leq j \leq n$;

(b) $\widehat{\mathbf{c}}^{j}=\widehat{\mathbf{h}}^{j}+\mathbf{B}^{j} \widehat{\mathbf{c}}^{j}, \quad 1 \leq j \leq n-1$.

The next result gives the required recursion between successive pivots.

\section{Lemma 4.14}

$$
q^{\alpha}(j) c^{\alpha, S(j+1)}(j)=\frac{c \mu+\lambda c^{\alpha, S(j)}(j-1)}{\alpha+\lambda+\mu}, \quad 1 \leq j \leq n-1 .
$$

Proof. Fix $1 \leq j \leq n-1$. By Lemma 4.13 and the definitions of $\mathbf{h}^{j}, \widehat{\mathbf{h}}^{j}$, we have

$$
\widehat{\mathbf{c}}^{j}-\mathbf{c}^{j}=\left(\mathbf{I}-\mathbf{B}^{j}\right)^{-1}\left(\widehat{\mathbf{h}}^{j}-\mathbf{h}^{j}\right)=\frac{\mu c^{\alpha, S(j+1)}(j)}{\alpha+\lambda+\mu}\left(\mathbf{I}-\mathbf{B}^{j}\right)^{-1} \mathbf{e}_{j} .
$$

Now, noting again that the element in position $(j, j)$ of matrix $\left(\mathbf{I}-\mathbf{B}^{j}\right)^{-1}$ is $1 / q^{\alpha}(j-1)$, it follows from (28) that

$$
c^{\alpha, S(j+1)}(j-1)-c^{\alpha, S(j)}(j-1)=\frac{\mu}{q^{\alpha}(j-1)} \frac{c^{\alpha, S(j+1)}(j)}{\alpha+\lambda+\mu} .
$$

We next substitute for $c^{\alpha, S(j+1)}(j-1)$ in (29) using Lemma 4.6's identity

$$
c^{\alpha, S(j+1)}(j)=\frac{c \mu}{\alpha+\lambda+\mu}+\frac{\lambda}{\alpha+\lambda+\mu} c^{\alpha, S(j+1)}(j-1),
$$

and further substitute for $q^{\alpha}(j-1)$ in terms of $q^{\alpha}(j)$ using Lemma 4.8(b), to obtain the required identity. This completes the proof. 


\subsubsection{Discounted MPI}

We next set out to establish PCL( $\mathscr{F})$-indexability and to calculate the discounted MPI. The following result gives a recursion for constructing index $\nu^{\alpha, *}(i) \triangleq c^{\alpha, S(i+1)}(i) / w^{\alpha, S(i+1)}(i)$, for $0 \leq i \leq n-1$ (cf. (11)), and further establishes the remarkable result that the index value does not depend on the buffer size $n$.

\section{Proposition 4.15}

(a) Index $\nu^{\alpha, *}(i)$ is calculated by the following recursion:

$$
\nu^{\alpha, *}(i)= \begin{cases}\frac{c+r \lambda}{\alpha+\lambda} \mu & \text { if } i=0 \\ \nu^{\alpha, *}(i-1)-\frac{\alpha \nu^{\alpha, *}(i-1)-c \mu}{\alpha+\lambda w^{\alpha, S(i)}(i-1)} & \text { if } 1 \leq i \leq n-1 .\end{cases}
$$

(b) For each $0 \leq i \leq n-1, \nu^{\alpha, *}(i)$ does not depend on the buffer size $n$.

Proof. (a) We have, by (22) and (27),

$$
\nu^{\alpha, *}(0) \triangleq \frac{c^{\alpha, S(1)}(0)}{w^{\alpha, S(1)}(0)}=\frac{c+r \lambda}{\alpha+\lambda} \mu .
$$

Further, for $1 \leq i \leq n-1$, using Lemma 4.9 and Lemma 4.14 we obtain that

$$
\begin{aligned}
\nu^{\alpha, *}(i) \triangleq \frac{c^{\alpha, S(i+1)}(i)}{w^{\alpha, S(i+1)}(i)} & =\frac{c \mu+\lambda c^{\alpha, S(i)}(i-1)}{\alpha+\lambda w^{\alpha, S(i)}(i-1)}=\frac{c \mu+\nu^{\alpha, *}(i-1) \lambda w^{\alpha, S(i)}(i-1)}{\alpha+\lambda w^{\alpha, S(i)}(i-1)} \\
& =\nu^{\alpha, *}(i-1)-\frac{\alpha \nu^{\alpha, *}(i-1)-c \mu}{\alpha+\lambda w^{\alpha, S(i)}(i-1)}
\end{aligned}
$$

(b) The result follows from part (a) and by noting that pivot marginal workloads $w^{\alpha, S(i)}(i-1)$ do not depend on the buffer size either. This completes the proof.

We will find it useful to reformulate the second identity in (30) as

$$
\alpha \nu^{\alpha, *}(i)-c \mu=\frac{\lambda w^{\alpha, S(i)}(i-1)}{\alpha+\lambda w^{\alpha, S(i)}(i-1)}\left\{\alpha \nu^{\alpha, *}(i-1)-c \mu\right\}, \quad 1 \leq i \leq n-1 .
$$

Proposition 4.16 The following inequalities hold (strictly iff $\alpha r>c$ ):

(a) $\alpha \nu^{\alpha, *}(i) \geq c \mu$, for $0 \leq i \leq n-1$.

(b) $\nu^{\alpha, *}(n-1) \leq \nu^{\alpha, *}(n-2) \leq \cdots \leq \nu^{\alpha, *}(0)$.

Proof. (a) We argue by upward induction on $i$. The case $i=0$ is easily seen to be equivalent to the assumed condition $\alpha r \geq c$. Suppose now the inequality holds for some $0 \leq i \leq n-2$. Then, (31) and Proposition 4.12(a) imply that $\alpha \nu^{\alpha, *}(i+1) \geq c \mu$, completing the induction proof.

(b) The result follows from part (a), Proposition 4.12(a), and identity (30).

The result that the inequalities are strict iff $\alpha r>c$ follows along the same lines.

We can now give the main result of this section.

\section{Theorem 4.17}

(a) An $\alpha$-discount loss-sensitive class is $\alpha$-discount PCL $(\mathscr{F})$-indexable with $\operatorname{MPI} \nu^{\alpha, *}(i)$, which satisfies

$$
\max _{i \in S \in \mathscr{F}} \nu^{\alpha, S}(i)=\nu^{\alpha, *}(i)=\min _{i \notin S \in \mathscr{F}} \nu^{\alpha, S}(i), \quad 0 \leq i \leq n-1 .
$$

(b) The class is $\alpha$-discount PCL( $\mathscr{F})$-indexable for any $\alpha>0$ iff it is pure loss-sensitive $(r>0=c)$.

Proof. (a) The result follows from Proposition 4.12(a) and Proposition 4.16(a). The stated characterization of the MPI follows fro Theorem 3.4.

(b) This part follows immediately from the above. 
Notice that, in light of Proposition 4.15(b), we can consider the sequence $\left\{\nu^{\alpha, *}(i): i \geq 0\right\}$. By Proposition 4.16, the latter is is monotone nonincreasing and bounded below, and hence it is convergent. The following result, which follows immediately from the above, gives its limit.

\section{Corollary 4.18}

$$
\lim _{i \rightarrow \infty} \nu^{\alpha, *}(i)=\frac{c \mu}{\alpha}
$$

\subsection{Average criterion: average and second-order MPI}

It is of interest to extend the above analysis to the average criterion, as outlined in Section 3.2, to obtain appropriate index policies for scheduling problem (3). In light of Theorem 4.17(b), we restrict attention in this Section to the pure loss-sensitive case $r>0=c$.

In short, it is readily seen that a pure loss-sensitive class is $\operatorname{PCL}(\mathscr{F})$-indexable relative to the average criterion. One thus obtains average-criterion counterparts to each result in Section 4.1 by letting $\alpha \searrow 0$. The resulting average MPI is

$$
\nu^{*}(i)=\lim _{\alpha \searrow 0} \nu^{\alpha, *}(i) \equiv r \mu, \quad 0 \leq i \leq n-1,
$$

so that it is constant across states.

To obtain a more informative, tie-breaking index, we proceed as in Section 3.2 to introduce the secondorder MPI, based on the McLaurin series expansion of the discounted MPI:

$$
\nu^{\alpha, *}(i)=r \mu-\alpha \gamma^{*}(i)+o(\alpha) \quad \text { as } \alpha \searrow 0 .
$$

We thus define the second-order MPI by

$$
\gamma^{*}(i) \triangleq \lim _{\alpha \searrow 0} \frac{r \mu-\nu^{\alpha, *}(i)}{\alpha}, \quad 0 \leq i \leq n-1
$$

We will obtain closed-form expressions for the second-order MPI. For such purpose, we will use coefficients

$$
q(i) \triangleq \lim _{\alpha \searrow 0} q^{\alpha}(i), \quad 0 \leq i \leq n-1
$$

and pivot average marginal workloads

$$
w^{S(i+1)}(i) \triangleq \lim _{\alpha \searrow 0} w^{\alpha, S(i+1)}(i), \quad 0 \leq i \leq n-1 .
$$

In both cases, it suffice to set $\alpha=0$ in the relevant results of Section 4.1.

Let us start with the $q(i)$ 's. Notice that in what follows we will write $\rho \triangleq \lambda / \mu$.

\section{Lemma 4.19}

(a) The $q(i)$ 's are calculated by the following recursion: $q(0)=1$, and

$$
q(i)=1-\frac{\rho}{(1+\rho)^{2} q(i-1)}, \quad 1 \leq i \leq n-1 .
$$

(b) The solution to such recursion is: for $0 \leq i \leq n-1$,

$$
q(i)=\frac{1}{1+\rho} \frac{1+\cdots+\rho^{i+1}}{1+\cdots+\rho^{i}}= \begin{cases}\frac{1}{1+\rho} \frac{1-\rho^{i+2}}{1-\rho^{i+1}} & \text { if } \rho \neq 1 \\ \frac{1}{2} \frac{i+2}{i+1} & \text { if } \rho=1 .\end{cases}
$$

Proof. Part (a) follows by Lemma 4.8(b). Part (b) follows by upward recursion.

The corresponding result for the $w^{S(i+1)}(i)$ 's follows.

\section{Lemma 4.20}


(a) The $w^{S(i+1)}(i)$ 's are calculated by the following recursion: $w^{S(1)}(0)=\rho /(1+\rho)$, and

$$
w^{S(i+1)}(i)=\frac{\rho}{1+\rho} \frac{w^{S(i)}(i-1)}{q(i)}, \quad 1 \leq i \leq n-1 .
$$

(b) The solution to such recursion is: for $0 \leq i \leq n-1$,

$$
w^{S(i+1)}(i)=\frac{\rho^{i+1}}{1+\cdots+\rho^{i+1}}= \begin{cases}(1-\rho) \frac{\rho^{i+1}}{1-\rho^{i+2}} & \text { if } \rho \neq 1 \\ \frac{1}{i+2} & \text { if } \rho=1 .\end{cases}
$$

Proof. Part (a) follows by Lemma 4.9. Part (b) follows by upward recursion.

We can now calculate the second-order MPI.

\section{Proposition 4.21}

(a) The second-order MPI is calculated by the following recursion: $\gamma^{*}(0)=r / \rho$, and

$$
\gamma^{*}(i)=\gamma^{*}(i-1)+\frac{r / \rho}{w^{S(i)}(i-1)}, \quad 1 \leq i \leq n-1
$$

(b) The solution to such recursion is: for $0 \leq i<n-1$,

$$
\gamma^{*}(i)= \begin{cases}\frac{r}{\rho}\left\{i+1+\frac{1 / \rho^{i}-(1-\rho) i-1}{(1-\rho)^{2}}\right\} & \text { if } \rho \neq 1 \\ r \frac{(i+1)(i+2)}{2} & \text { if } \rho=1 .\end{cases}
$$

Proof. Part (a) follows by substituting for discounted MPI $\nu^{\alpha, *}(i)$ in recursion (30) the McLaurin expansion (33), and then letting $\alpha$ vanish.

Part (b) is readily verified by induction, drawing on Lemma 4.20(b).

Notice that the second-order MPI is monotonically increasing in the number of empty buffer spaces:

$$
\gamma^{*}(0)<\gamma^{*}(1)<\cdots<\gamma^{*}(n-1)
$$

\section{PCL-indexability analysis: delay-sensitive classes}

We address in this section the PCL-indexability analysis for a delay-sensitive class in isolation, i.e. an $M / M / 1 / n$ queue with arrival and service rates $\lambda$ and $\mu$, respectively, subject to service control, with delay cost rate $c>0$ and rejection cost rate $r \geq 0$ satisfying $c \geq \alpha r$.

For such a class, we define the state by $\bar{X}(t) \triangleq L(t)$, the number of jobs in system. We will use the state

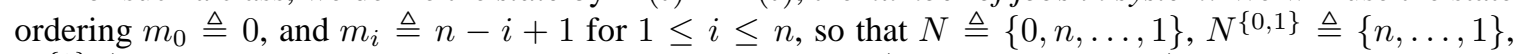
$N^{\{0\}} \triangleq\{0\}$, and the active-state sets in $\mathscr{F}$ are given by $S(0) \triangleq\{n, \ldots, 1\}, S(1) \triangleq \emptyset$, and

$$
S(i) \triangleq\{i-1, \ldots, 1\}, \quad i=2, \ldots, n
$$

In words, $\mathscr{F}$-policies prescribe the server to work when the number of jobs in system is small enough.

For notational convenience we will write henceforth $S(0)$ as $S(n+1)$. 


\subsection{Discounted criterion}

We start by laying the groundwork for calculations of discounted marginal workloads and costs, using uniformization as before with rate $\Lambda \geq \lambda+\mu$. The uniformized MDP's state transition probability matrix $\mathbf{P}^{a}=\left(p_{i j}^{a}\right)$ under action $a \in\{0,1\}$ is given by

$$
p_{i j}^{a} \triangleq \begin{cases}\lambda / \Lambda & \text { if } 1 \leq j=i+1 \leq n \\ \mu a / \Lambda & \text { if } 0 \leq j=i-1 \leq n-1 \\ (\Lambda-\lambda-\mu a) / \Lambda & \text { if } 0<j=i<n \\ (\Lambda-\lambda) / \Lambda & \text { if } j=i=0 \\ (\Lambda-\mu a) / \Lambda & \text { if } j=i=n \\ 0 & \text { otherwise. }\end{cases}
$$

The corresponding discrete-time discount factor is $\Lambda /(\alpha+\Lambda)$, and the discrete-time one-period cost rate in state $i$ is $\left\{c i+r \lambda 1_{\{i=n\}}\right\} /(\alpha+\Lambda)$.

Consider now discounted marginal workloads $w^{\alpha, S}(i)$, for $i \in N^{\{0,1\}}$ and $S \in \mathscr{F}$. From their definition in (8) and uniformization we readily obtain

$$
w^{\alpha, S}(i)=1-\mu \Delta g^{\alpha, S}(i),
$$

where $\Delta g^{\alpha, S}(i) \triangleq g^{\alpha, S}(i)-g^{\alpha, S}(i-1)$.

We thus start by giving the evaluation equations for the $g^{\alpha, S}(i)$ 's, for fixed $S \in \mathscr{F}$. We will denote by $\mu_{S}(i)$, as before, the effective service rate in state $i$ under the $S$-active policy, i.e. letting $1_{S}(i)$ be the indicator function of $S$,

$$
\mu_{S}(i) \triangleq \mu 1_{S}(i), \quad i \in N
$$

Lemma 5.1 Discounted work measures $g^{\alpha, S}(i)$, for $i \in N$, are characterized by the equations

$$
\begin{aligned}
\alpha g^{\alpha, S}(0) & =\lambda \Delta g^{\alpha, S}(1) \\
\alpha g^{\alpha, S}(i) & =1_{S}(i)-\mu_{S}(i) \Delta g^{\alpha, S}(i)+\lambda \Delta g^{\alpha, S}(i+1), \quad 1 \leq i \leq n-1 \\
\alpha g^{\alpha, S}(n) & =1_{S}(n)-\mu_{S}(n) \Delta g^{\alpha, S}(n) .
\end{aligned}
$$

The next result, characterizing first-order differences $\Delta g^{\alpha, S}(i)$, follows immediately.

Lemma 5.2 Terms $\Delta g^{\alpha, S}(i)$, for $i \in N^{\{0,1\}}$, are characterized by the equations

$$
\begin{aligned}
\left(\alpha+\lambda+\mu_{S}(1)\right) \Delta g^{\alpha, S}(1) & =1_{S}(1)+\lambda \Delta g^{\alpha, S}(2) \\
\left(\alpha+\lambda+\mu_{S}(i)\right) \Delta g^{\alpha, S}(i) & =\Delta 1_{S}(i)+\mu_{S}(i-1) \Delta g^{\alpha, S}(i-1)+\lambda \Delta g^{\alpha, S}(i+1), \quad 2 \leq i \leq n-1 \\
\left(\alpha+\lambda+\mu_{S}(n)\right) \Delta g^{\alpha, S}(n) & =\Delta 1_{S}(n)+\mu_{S}(n-1) \Delta g^{\alpha, S}(n-1) .
\end{aligned}
$$

We can now give the evaluation equations for discounted marginal workloads.

Lemma 5.3 Discounted marginal workloads $w^{\alpha, S}(i)$, for $i \in N^{\{0,1\}}$, are characterized by the equations

$$
\begin{aligned}
\left(\alpha+\lambda+\mu_{S}(1)\right) w^{\alpha, S}(1) & =\alpha+\lambda w^{\alpha, S}(2) \\
\left(\alpha+\lambda+\mu_{S}(i)\right) w^{\alpha, S}(i) & =\alpha+\mu_{S}(i-1) w^{\alpha, S}(i-1)+\lambda w^{\alpha, S}(i+1), \quad 2 \leq i \leq n-1 \\
\left(\alpha+\lambda+\mu_{S}(n)\right) w^{\alpha, S}(n) & =\alpha+\lambda+\mu_{S}(n-1) w^{\alpha, S}(n-1) .
\end{aligned}
$$

Proof. The result follows immediately from identity (35) and Lemma 5.2.

We next turn attention to discounted marginal costs $c^{\alpha, S}(i)$, for $i \in N^{\{0,1\}}$ and $S \in \mathscr{F}$. From their definition in (9) and uniformization we readily obtain

$$
c^{\alpha, S}(i)=\mu \Delta f^{\alpha, S}(i)
$$

Proceeding as before, we next state the standard evaluation equations for the $f^{\alpha, S}(i)$ 's. 
Lemma 5.4 Discounted cost measures $f^{\alpha, S}(i)$, for $i \in N$, are characterized by the equations

$$
\begin{aligned}
\alpha f^{\alpha, S}(0) & =\lambda \Delta f^{\alpha, S}(1) \\
\alpha f^{\alpha, S}(i) & =c i-\mu_{S}(i) \Delta f^{\alpha, S}(i)+\lambda \Delta f^{\alpha, S}(i+1), 1 \leq i \leq n-1 \\
\alpha f^{\alpha, S}(n) & =c n+r \lambda-\mu_{S}(n) \Delta f^{\alpha, S}(n) .
\end{aligned}
$$

The next result, characterizing first-order differences $\Delta f^{\alpha, S}(i)$, follows immediately.

Lemma 5.5 Terms $\Delta f^{\alpha, S}(i)$, for $i \in N^{\{0,1\}}$, are characterized by the equations

$$
\begin{aligned}
\left(\alpha+\lambda+\mu_{S}(1)\right) \Delta f^{\alpha, S}(1) & =c+\lambda \Delta f^{\alpha, S}(2) \\
\left(\alpha+\lambda+\mu_{S}(i)\right) \Delta f^{\alpha, S}(i) & =c+\mu_{S}(i-1) \Delta f^{\alpha, S}(i-1)+\lambda \Delta f^{\alpha, S}(i+1), 2 \leq i<n \\
\left(\alpha+\lambda+\mu_{S}(n)\right) \Delta f^{\alpha, S}(n) & =c+r \lambda+\mu_{S}(n-1) \Delta f^{\alpha, S}(n-1) .
\end{aligned}
$$

We can now give the evaluation equations for discounted marginal costs.

Lemma 5.6 Discounted marginal costs $c^{\alpha, S}(i)$, for $i \in N^{\{0,1\}}$, are characterized by the equations

$$
\begin{aligned}
\left(\alpha+\lambda+\mu_{S}(1)\right) c^{\alpha, S}(1) & =c \mu+\lambda c^{\alpha, S}(2) \\
\left(\alpha+\lambda+\mu_{S}(i)\right) c^{\alpha, S}(i) & =c \mu+\mu_{S}(i-1) c^{\alpha, S}(i-1)+\lambda c^{\alpha, S}(i+1), 2 \leq i \leq n-1 \\
\left(\alpha+\lambda+\mu_{S}(n)\right) c^{\alpha, S}(n) & =(c+r \lambda) \mu+\mu_{S}(n-1) c^{\alpha, S}(n-1) .
\end{aligned}
$$

Proof. The result follows immediately from identity (36) and Lemma 5.5.

\subsubsection{Discounted marginal workloads: calculation and properties}

We next set out to calculate discounted marginal workloads, and to establish their required properties.

We will develop a recursion to solve the evaluation equations in Lemma 5.3 for every active-state set $S(j)$, with $1 \leq j \leq n+1$ (recall that we write $S(n+1)=S(0)=N^{\{0,1\}}$ ). Notice that the case $j=1$ is trivial, since $S(1)=\emptyset$ and hence $w^{S(1)}(i) \equiv 1$. For other cases, calculations will proceed by upward recursion on $j$. We start with the equations for $j=2$,

$$
\begin{aligned}
(\alpha+\lambda+\mu) w^{\alpha, S(2)}(1) & =\alpha+\lambda w^{\alpha, S(2)}(2) \\
(\alpha+\lambda) w^{\alpha, S(2)}(2) & =\alpha+\mu w^{\alpha, S(2)}(1)+\lambda w^{\alpha, S(2)}(3) \\
(\alpha+\lambda) w^{\alpha, S(2)}(i) & =\alpha+\lambda w^{\alpha, S(2)}(i+1), \quad 3 \leq i \leq n-1 \\
(\alpha+\lambda) w^{\alpha, S(2)}(n) & =\alpha+\lambda,
\end{aligned}
$$

whose solution is

$$
\begin{aligned}
& w^{\alpha, S(2)}(1)=\frac{(\alpha+\lambda)^{2}}{(\alpha+\lambda)^{2}+\alpha \mu}, \quad w^{\alpha, S(2)}(2)=1+\mu \frac{\alpha+\lambda}{(\alpha+\lambda)^{2}+\alpha \mu} \\
& w^{\alpha, S(2)}(i)=1, \quad 3 \leq i \leq n .
\end{aligned}
$$

Notice that it suffices to know the value of pivot term $w^{\alpha, S(2)}(2)$, from which remaining terms $w^{\alpha, S(2)}(i)$ are readily calculated. Similarly, if for a given $2 \leq j \leq n$ pivot term $w^{\alpha, S(j)}(j)$ were available, from the remaining equations for $S(j)$ we could readily calculate remaining terms $w^{\alpha, S(j)}(i)$. Noting further that

$$
w^{\alpha, S(j)}(i)=1, \quad j+1 \leq i \leq n .
$$

Thus, if we could represent pivot $w^{\alpha, S(j+1)}(j+1)$ in terms of previous pivot $w^{\alpha, S(j)}(j)$, for $2 \leq j \leq n-1$, such relations would furnish the backbone of a recursion to calculate all marginal workloads $w^{\alpha, S(j)}(i)$ for $2 \leq j \leq n$. To complete the calculations, we will need a further relation between pivot $w^{\alpha, S(n)}(n)$ and the last pivot, which we take to be $w^{\alpha, S(n+1)}(n)$. Again, from the latter we easily obtain remaining terms $w^{\alpha, S(n+1)}(i)$. 
We next set out to relate successive pivots, along the lines followed before in the loss-sensitive case. We will use the following vectors: for $2 \leq j \leq n+1$, let

$$
\begin{aligned}
& \mathbf{w}^{j} \triangleq\left[\begin{array}{ll}
w^{\alpha, S(j)}(1) \quad \cdots \quad w^{\alpha, S(j)}(j-1)
\end{array}\right]^{\mathrm{T}} \\
& \mathbf{b}^{j} \triangleq \begin{cases}\frac{\alpha}{\alpha+\lambda+\mu} \mathbf{1}+\frac{\lambda w^{\alpha, S(j)}(j)}{\alpha+\lambda+\mu} \mathbf{e}_{j-1} & \text { if } 2 \leq j \leq n \\
\frac{\alpha}{\alpha+\lambda+\mu} \mathbf{1}+\frac{\lambda}{\alpha+\lambda+\mu} \mathbf{e}_{n} & \text { if } j=n+1 ;\end{cases}
\end{aligned}
$$

and, for $2 \leq j \leq n$, let

$$
\begin{aligned}
& \widehat{\mathbf{w}}^{j} \triangleq\left[\begin{array}{lll}
w^{\alpha, S(j+1)}(1) & \cdots & w^{\alpha, S(j+1)}(j-1)
\end{array}\right]^{\mathrm{T}} \\
& \widehat{\mathbf{b}}^{j} \triangleq \mathbf{b}^{j}+\lambda\left\{\frac{w^{\alpha, S(j+1)}(j)-w^{\alpha, S(j)}(j)}{\alpha+\lambda+\mu}\right\} \mathbf{e}_{j-1} .
\end{aligned}
$$

Let us further introduce, for $2 \leq j \leq n+1$, the square matrix of dimension $j-1$

$$
\mathbf{B}^{j} \triangleq \frac{1}{\alpha+\lambda+\mu}\left[\begin{array}{ccccc}
0 & \lambda & & & \\
\mu & 0 & \lambda & & \\
& \ddots & \ddots & \ddots & \\
& & \mu & 0 & \lambda \\
& & & \mu & 0
\end{array}\right],
$$

with $\mathbf{B}^{2} \triangleq 0$. The next result reformulates some equations in Lemma 5.3.

\section{Lemma 5.7}

(a) $\mathbf{w}^{j}=\mathbf{b}^{j}+\mathbf{B}^{j} \mathbf{w}^{j}, \quad 2 \leq j \leq n+1$.

(b) $\widehat{\mathbf{w}}^{j}=\widehat{\mathbf{b}}^{j}+\mathbf{B}^{j} \widehat{\mathbf{w}}^{j}, \quad 2 \leq j \leq n$.

To proceed, we introduce coefficients $q^{\alpha}(2), \ldots, q^{\alpha}(n+1)$, defined by

$$
q^{\alpha}(j)= \begin{cases}1 & \text { if } j=2 \\ \frac{\operatorname{det}\left(\mathbf{I}-\mathbf{B}^{j}\right)}{\operatorname{det}\left(\mathbf{I}-\mathbf{B}^{j-1}\right)} & \text { if } 3 \leq j \leq n+1 .\end{cases}
$$

The following result is equivalent to Lemma 4.8.

Lemma 5.8 Terms $q^{\alpha}(j)$ are well defined, and satisfy the following properties:

(a) $q^{\alpha}(j)>0$, for $2 \leq j \leq n+1$.

(b) They can be computed by upward recursion, setting $q^{\alpha}(2)=1$ and

$$
q^{\alpha}(j)=1-\frac{\lambda \mu}{(\alpha+\lambda+\mu)^{2} q^{\alpha}(j-1)}, \quad 3 \leq j \leq n+1 .
$$

(c) $\frac{\alpha+\mu}{\alpha+\lambda+\mu}<q^{\alpha}(j)<1, \quad 3 \leq j \leq n+1$.

We are now ready to relate successive pivots.

\section{Lemma 5.9}

$w^{\alpha, S(j+1)}(j+1)=1+\mu \frac{(\alpha+\lambda+\mu) q^{\alpha}(j+1)-\mu}{(\alpha+\lambda)(\alpha+\lambda+\mu) q^{\alpha}(j+1)-\lambda \mu} w^{\alpha, S(j)}(j), \quad 2 \leq j \leq n-1$,

and

$$
w^{\alpha, S(n+1)}(n)=\frac{(\alpha+\lambda+\mu) q^{\alpha}(n+1)-\mu}{(\alpha+\lambda+\mu) q^{\alpha}(n+1)} w^{\alpha, S(n)}(n)
$$


Proof. Fix $2 \leq j \leq n-1$. By Lemma 5.7, we have

$$
\widehat{\mathbf{w}}^{j}-\mathbf{w}^{j}=\left(\mathbf{I}-\mathbf{B}^{j}\right)^{-1}\left(\widehat{\mathbf{b}}^{j}-\mathbf{b}^{j}\right)=\frac{\lambda\left\{w^{\alpha, S(j+1)}(j)-w^{\alpha, S(j)}(j)\right\}}{\alpha+\lambda+\mu}\left(\mathbf{I}-\mathbf{B}^{j}\right)^{-1} \mathbf{e}_{j-1} .
$$

Now, noting that the element in position $(j-1, j-1)$ of matrix $\left(\mathbf{I}-\mathbf{B}^{j}\right)^{-1}$ is $\operatorname{det}\left(\mathbf{I}-\mathbf{B}^{j-1}\right) / \operatorname{det}\left(\mathbf{I}-\mathbf{B}^{j}\right)$, which by definition equals $1 / q^{\alpha}(j)$, it follows from (40) that

$$
w^{\alpha, S(j+1)}(j-1)-w^{\alpha, S(j)}(j-1)=\frac{\lambda}{q^{\alpha}(j)} \frac{\left\{w^{\alpha, S(j+1)}(j)-w^{\alpha, S(j)}(j)\right\}}{\alpha+\lambda+\mu} .
$$

We further have the equations (cf. Lemma 5.3 and (38)

$$
\begin{aligned}
(\alpha+\lambda) w^{\alpha, S(j)}(j) & =\alpha+\lambda+\mu w^{\alpha, S(j)}(j-1) \\
(\alpha+\lambda+\mu) w^{\alpha, S(j+1)}(j) & =\alpha+\mu w^{\alpha, S(j+1)}(j-1)+\lambda w^{\alpha, S(j+1)}(j+1) \\
(\alpha+\lambda) w^{\alpha, S(j+1)}(j+1) & =\alpha+\lambda+\mu w^{\alpha, S(j+1)}(j) .
\end{aligned}
$$

Now, from (41)-(42) and Lemma 5.8(b) we obtain

$$
w^{\alpha, S(j+1)}(j+1)=1+\mu \frac{(\alpha+\lambda+\mu) q^{\alpha}(j+1)-\mu}{(\alpha+\lambda)(\alpha+\lambda+\mu) q^{\alpha}(j+1)-\lambda \mu} w^{\alpha, S(j)}(j),
$$

as required.

To obtain the relation between the last two pivots, we use the equations

$$
\begin{aligned}
w^{\alpha, S(n+1)}(n-1)-w^{\alpha, S(n)}(n-1) & =\frac{\lambda}{q^{\alpha}(n)} \frac{\left\{w^{\alpha, S(n+1)}(n)-w^{\alpha, S(n)}(n)\right\}}{\alpha+\lambda+\mu} \\
(\alpha+\lambda) w^{\alpha, S(n)}(n) & =\alpha+\lambda+\mu w^{\alpha, S(n)}(n-1) \\
(\alpha+\lambda+\mu) w^{\alpha, S(n+1)}(n) & =\alpha+\lambda+\mu w^{\alpha, S(n+1)}(n-1),
\end{aligned}
$$

from which we get, using again Lemma 5.8(b),

$$
w^{\alpha, S(n+1)}(n)=\frac{(\alpha+\lambda+\mu) q^{\alpha}(n+1)-\mu}{(\alpha+\lambda+\mu) q^{\alpha}(n+1)} w^{\alpha, S(n)}(n) .
$$

This completes the proof.

We can now give a complete recursion for calculating discounted marginal workloads, as shown in Figure 3. Figure 4 further clarifies the recursion, showing by arrows the directions in which calculations proceed, and enclosing in boxes the pivot terms.

Proposition 5.10 Discounted marginal workloads $w^{\alpha, S}(i)$, for $i \in N^{\{0,1\}}$ and $S \in \mathscr{F}$, are calculated by the recursion shown in Figure 3.

Proof. The result follows directly from Lemmas 5.3 and 5.9.

We next use the above to establish required properties of discounted marginal workloads. Figure 4 illustrates the inequalities presented in the following result.

Lemma 5.11 Discounted marginal workloads satisfy the following inequalities:

(a) $w^{\alpha, S(j)}(j)>1$, for $2 \leq j \leq n$, and $w^{\alpha, S(n+1)}(n)>0$.

(b) $w^{\alpha, S(j)}(i)>w^{\alpha, S(j+1)}(i)$, for $2 \leq j \leq n$ and $1 \leq i \leq j$.

(c) $w^{\alpha, S(j)}(i)=1$, for $1 \leq j \leq n-1$ and $j+1 \leq i \leq n$.

(d) $w^{\alpha, S(n+1)}(i)>0, \quad 1 \leq i \leq n$. 
Calculation of $w^{\alpha, S(1)}(i)$ 's: $w^{\alpha, S(1)}(i)=1, \quad 1 \leq i \leq n$

Calculation of $w^{\alpha, S(2)}(i)$ 's: $w^{\alpha, S(2)}(i)=1, \quad 3 \leq i \leq n$

$$
w^{\alpha, S(2)}(2)=1+\mu \frac{\alpha+\lambda}{(\alpha+\lambda)^{2}+\alpha \mu} ; \quad w^{\alpha, S(2)}(1)=\frac{\alpha+\lambda w^{\alpha, S(2)}(2)}{\alpha+\lambda+\mu}
$$

Calculation of $w^{\alpha, S(j)}(i)$ 's, for $j=3$ to $n: w^{\alpha, S(j)}(i)=1, \quad j+1 \leq i \leq n$

$$
\begin{aligned}
w^{\alpha, S(j)}(j) & =1+\mu \frac{(\alpha+\lambda+\mu) q^{\alpha}(j)-\mu}{(\alpha+\lambda)(\alpha+\lambda+\mu) q^{\alpha}(j)-\lambda \mu} w^{\alpha, S(j-1)}(j-1) \\
w^{\alpha, S(j)}(j-1) & =\frac{\alpha+\lambda}{\mu}\left\{w^{\alpha, S(j)}(j)-1\right\} \\
w^{\alpha, S(j)}(i) & =\frac{-\alpha+(\alpha+\lambda+\mu) w^{\alpha, S(j)}(i+1)-\lambda w^{\alpha, S(j)}(i+2)}{\mu}, \quad 1 \leq i \leq j-2
\end{aligned}
$$

\begin{tabular}{|c|c|c|c|c|c|c|c|c|}
\hline 1 & $>$ & $\begin{array}{c}w^{\alpha, S(2)}(1) \\
\uparrow\end{array}$ & $>$ & $\ldots$ & $>$ & $\begin{array}{c}w^{S(n)}(1) \\
\uparrow\end{array}$ & $>$ & $\begin{array}{c}w^{\alpha, S(n+1)}(1) \\
\uparrow\end{array}$ \\
\hline 1 & $<$ & $w^{\alpha, S(2)}(2)$ & $>$ & . & $>$ & $w^{\alpha, S(n)}(2)$ & $>$ & $w^{\alpha, S(n+1)}(2)$ \\
\hline 1 & & 1 & $\begin{array}{l}\searrow \\
<\end{array}$ & $\ldots$ & $>$ & $w^{\alpha, S(n)}(3)$ & $>$ & $\begin{array}{c}\uparrow \\
w^{\alpha, S(n+1)}(3)\end{array}$ \\
\hline$\vdots$ & & $\vdots$ & & $\ddots$ & 7 & $\begin{array}{l}\vdots \\
\uparrow\end{array}$ & & $\begin{array}{l}\vdots \\
\uparrow\end{array}$ \\
\hline 1 & & 1 & 1 & $\ldots$ & $<$ & $w^{\alpha, S(n)}(n)$ & $>\rightarrow$ & $w^{\alpha, S(n+1)}(n)$ \\
\hline
\end{tabular}

Calculation of $w^{\alpha, S(n+1)}(i)$ 's:

$$
\begin{aligned}
w^{\alpha, S(n+1)}(n) & =\frac{(\alpha+\lambda+\mu) q^{\alpha}(n+1)-\mu}{(\alpha+\lambda+\mu) q^{\alpha}(n+1)} w^{\alpha, S(n)}(n) \\
w^{\alpha, S(n+1)}(n-1) & =\frac{-(\alpha+\mu)+(\alpha+\lambda+\mu) w^{\alpha, S(n+1)}(n)}{\mu} \\
w^{\alpha, S(n+1)}(i) & =\frac{-\alpha+(\alpha+\lambda+\mu) w^{\alpha, S(n+1)}(i+1)-\lambda w^{\alpha, S(n+1)}(i+2)}{\mu}, 1 \leq i \leq n-2
\end{aligned}
$$

Figure 3: Marginal workloads: recursive calculation.

Figure 4: Marginal workloads: directions of calculations and inequalities. 
Proof. (a) This part follows by upward induction on $j$, via (37) and Lemmas 5.8(a) and 5.9.

(b) Fix $2 \leq j \leq n$. In the case $2 \leq j \leq n-1$, we have

$$
\begin{aligned}
\mathbf{w}^{j}-\widehat{\mathbf{w}}^{j} & =\frac{\lambda\left\{w^{\alpha, S(j)}(j)-w^{\alpha, S(j+1)}(j)\right\}}{\alpha+\lambda+\mu}\left(\mathbf{I}-\mathbf{B}^{j}\right)^{-1} \mathbf{e}_{j-1} \\
& =\frac{\alpha \lambda \mu w^{\alpha, S(j)}(j)}{(\alpha+\lambda+\mu)\left((\alpha+\lambda)(\alpha+\lambda+\mu) q^{\alpha}(j+1)-\lambda \mu\right)}\left(\mathbf{I}-\mathbf{B}^{j}\right)^{-1} \mathbf{e}_{j-1} \\
& >\mathbf{0}
\end{aligned}
$$

where we have used Lemma 5.7, the identity (which follows readily from (41)-(42))

$$
w^{\alpha, S(j+1)}(j)-w^{\alpha, S(j)}(j)=-\frac{\alpha \mu}{(\alpha+\lambda)(\alpha+\lambda+\mu) q^{\alpha}(j+1)-\lambda \mu} w^{\alpha, S(j)}(j),
$$

Lemma 5.8(c) and part (a).

Arguing similarly, in the case $j=n$ we have

$$
\begin{aligned}
\mathbf{w}^{n}-\widehat{\mathbf{w}}^{n} & =\frac{\lambda\left\{w^{\alpha, S(n)}(n)-w^{\alpha, S(n+1)}(n)\right\}}{\alpha+\lambda+\mu}\left(\mathbf{I}-\mathbf{B}^{n}\right)^{-1} \mathbf{e}_{n-1} \\
& =\frac{\lambda \mu w^{\alpha, S(n)}(n)}{(\alpha+\lambda+\mu)^{2} q^{\alpha}(n+1)}\left(\mathbf{I}-\mathbf{B}^{n}\right)^{-1} \mathbf{e}_{n-1} \\
& >\mathbf{0}
\end{aligned}
$$

where we have used the identity

$$
w^{\alpha, S(n+1)}(n)-w^{\alpha, S(n)}(n)=-\frac{\mu}{(\alpha+\lambda+\mu) q^{\alpha}(n+1)} w^{\alpha, S(n)}(n) .
$$

We have thus shown that

$$
w^{\alpha, S(j)}(i)>w^{\alpha, S(j+1)}(i), \quad 1 \leq i \leq j-1 .
$$

Further, we obtain from (41) and the inequalities just proven that $w^{\alpha, S(j)}(j)>w^{\alpha, S(j+1)}(j)$, as required.

(c) This part follows immediately from the evaluation equations.

(d) We have

$$
\mathbf{w}^{n+1}=\left(\mathbf{I}-\mathbf{B}^{n+1}\right)^{-1} \mathbf{b}^{n+1}>\mathbf{0},
$$

as required. This completes the proof.

We can now give the main result on discounted marginal workloads

Proposition 5.12 Discounted marginal workloads $w^{\alpha, S}(i)$ are positive, for $i \in N^{\{0,1\}}$ and $S \in \mathscr{F}$.

Proof. The result follows immediately from the inequalities and identities in Lemma 5.11, as illustrated in Figure 4.

\subsubsection{Discounted marginal costs: calculation}

We next set out to calculate the required discounted marginal costs $c^{\alpha, S}(i)$, proceeding similarly as before for discounted marginal workloads. Only pivot terms $c^{\alpha, S(j)}(j)$, for $1 \leq j \leq n$, are required for the PCLindexability analysis. We next develop a recursion to calculate them, along the lines followed above to calculate the $w^{\alpha, S(j)}(j)$ 's.

We start by formulating the system of evaluation equations in Lemma 5.6 for $j=1$ :

$$
\begin{aligned}
(\alpha+\lambda) c^{\alpha, S(1)}(i) & =c \mu+\lambda c^{\alpha, S(1)}(i+1), \quad 1 \leq i \leq n-1 \\
(\alpha+\lambda) c^{\alpha, S(1)}(n) & =(c+r \lambda) \mu .
\end{aligned}
$$

We thus obtain the first pivot, as

$$
c^{\alpha, S(1)}(1)=\frac{c \mu}{\alpha}\left\{1-\left(\frac{\lambda}{\alpha+\lambda}\right)^{n}\right\}+r \mu\left(\frac{\lambda}{\alpha+\lambda}\right)^{n} .
$$


The corresponding system of evaluation equations for $j=2$ is

$$
\begin{aligned}
(\alpha+\lambda+\mu) c^{S(2)}(1) & =c \mu+\lambda c^{S(2)}(2) \\
(\alpha+\lambda) c^{S(2)}(2) & =c \mu+\mu c^{S(2)}(1)+\lambda c^{S(2)}(3) \\
(\alpha+\lambda) c^{S(2)}(i) & =c \mu+\lambda c^{S(2)}(i+1), \quad 3 \leq i \leq n-1 \\
(\alpha+\lambda) c^{S(2)}(n) & =(c+r \lambda) \mu,
\end{aligned}
$$

whence we obtain the second pivot

$$
c^{S(2)}(2)=\frac{\alpha+\lambda+2 \mu}{(\alpha+\lambda)^{2}+\alpha \mu} c \mu+\frac{\lambda(\alpha+\lambda+\mu)}{(\alpha+\lambda)^{2}+\alpha \mu} c^{S(2)}(3),
$$

where we use the auxiliary term

$$
c^{\alpha, S(2)}(3)=\frac{c \mu}{\alpha}\left\{1-\left(\frac{\lambda}{\alpha+\lambda}\right)^{n-2}\right\}+r \mu\left(\frac{\lambda}{\alpha+\lambda}\right)^{n-2} .
$$

The last identity is easily seen to extend to

$$
c^{\alpha, S(j)}(j+1)=\frac{c \mu}{\alpha}\left\{1-\left(\frac{\lambda}{\alpha+\lambda}\right)^{n-j}\right\}+r \mu\left(\frac{\lambda}{\alpha+\lambda}\right)^{n-j}, \quad 1 \leq j \leq n-1,
$$

so that we have the downward recursion on auxiliary terms

$$
\begin{aligned}
c^{\alpha, S(n-1)}(n) & =\frac{c \mu+r \lambda \mu}{\alpha+\lambda} \\
c^{\alpha, S(j)}(j+1) & =\frac{c \mu}{\alpha+\lambda}+\frac{\lambda}{\alpha+\lambda} c^{\alpha, S(j+1)}(j+2), \quad 1 \leq j \leq n-2 .
\end{aligned}
$$

We next set out to relate successive pivots. Define the following vectors. For $2 \leq j \leq n$, let

$$
\begin{gathered}
\mathbf{c}^{j} \triangleq\left[\begin{array}{lll}
c^{\alpha, S(j)}(1) & \cdots & c^{\alpha, S(j)}(j-1)
\end{array}\right]^{\mathrm{T}}, \quad \mathbf{h}^{j} \triangleq \frac{c \mu}{\alpha+\lambda+\mu} \mathbf{1}+\frac{\lambda c^{\alpha, S(j)}(j)}{\alpha+\lambda+\mu} \mathbf{e}_{j-1}, \\
\widehat{\mathbf{c}}^{j} \triangleq\left[\begin{array}{lll}
c^{\alpha, S(j+1)}(1) & \cdots & c^{\alpha, S(j+1)}(j-1)
\end{array}\right]^{\mathrm{T}}, \quad \widehat{\mathbf{h}}^{j} \triangleq \mathbf{h}^{j}+\lambda\left\{\frac{c^{\alpha, S(j+1)}(j)-c^{\alpha, S(j)}(j)}{\alpha+\lambda+\mu}\right\} \mathbf{e}_{j-1} .
\end{gathered}
$$

The next result reformulates some equations in Lemma 5.6.

Lemma 5.13 For $2 \leq j \leq n$ :

(a) $\mathbf{c}^{j}=\mathbf{h}^{j}+\mathbf{B}^{j} \mathbf{c}^{j}$.

(b) $\widehat{\mathbf{c}}^{j}=\widehat{\mathbf{h}}^{j}+\mathbf{B}^{j} \widehat{\mathbf{c}}^{j}$.

We are now ready to establish the required recursion on pivot terms.

\section{Lemma 5.14}

$$
c^{\alpha, S(j+1)}(j+1)=c^{\alpha, S(j)}(j+1)+\mu \frac{(\alpha+\lambda+\mu) q^{\alpha}(j+1)-\mu}{(\alpha+\lambda)(\alpha+\lambda+\mu) q^{\alpha}(j+1)-\lambda \mu} c^{\alpha, S(j)}(j), \quad 2 \leq j \leq n-1 .
$$

Proof. Fix $2 \leq j \leq n-1$. By Lemma 5.13, we have

$$
\widehat{\mathbf{c}}^{j}-\mathbf{c}^{j}=\left(\mathbf{I}-\mathbf{B}^{j}\right)^{-1}\left(\widehat{\mathbf{h}}^{j}-\mathbf{h}^{j}\right)=\frac{\lambda\left\{c^{\alpha, S(j+1)}(j)-c^{\alpha, S(j)}(j)\right\}}{\alpha+\lambda+\mu}\left(\mathbf{I}-\mathbf{B}^{j}\right)^{-1} \mathbf{e}_{j-1} .
$$

Now, noting that the element in position $(j-1, j-1)$ of matrix $\left(\mathbf{I}-\mathbf{B}^{j}\right)^{-1}$ is $\operatorname{det}\left(\mathbf{I}-\mathbf{B}^{j-1}\right) / \operatorname{det}\left(\mathbf{I}-\mathbf{B}^{j}\right)$, which by definition equals $1 / q^{\alpha}(j)$, it follows from (47) that

$$
c^{\alpha, S(j+1)}(j-1)-c^{\alpha, S(j)}(j-1)=\frac{1}{q^{\alpha}(j)} \frac{\lambda\left\{c^{\alpha, S(j+1)}(j)-c^{\alpha, S(j)}(j)\right\}}{\alpha+\lambda+\mu} .
$$


We further have, in the case $j \leq n-2$, the equations (cf. Lemma 4.6)

$$
\begin{aligned}
(\alpha+\lambda) c^{\alpha, S(j)}(j) & =c \mu+\mu c^{\alpha, S(j)}(j-1)+\lambda c^{\alpha, S(j)}(j+1) \\
(\alpha+\lambda+\mu) c^{\alpha, S(j+1)}(j) & =c \mu+\mu c^{\alpha, S(j+1)}(j-1)+\lambda c^{\alpha, S(j+1)}(j+1) \\
(\alpha+\lambda) c^{\alpha, S(j+1)}(j+1) & =c \mu+\mu c^{\alpha, S(j+1)}(j)+\lambda c^{\alpha, S(j+1)}(j+2) .
\end{aligned}
$$

From the last four identities, (46) and Lemma 5.8(b), we obtain

$$
c^{\alpha, S(j+1)}(j+1)=c^{\alpha, S(j)}(j+1)+\mu \frac{(\alpha+\lambda+\mu) q^{\alpha}(j+1)-\mu}{(\alpha+\lambda)(\alpha+\lambda+\mu) q^{\alpha}(j+1)-\lambda \mu} c^{\alpha, S(j)}(j) .
$$

In the case $j=n-1$, we have the equations

$$
\begin{aligned}
(\alpha+\lambda) c^{\alpha, S(n-1)}(n-1) & =c \mu+\mu c^{\alpha, S(n-1)}(n-2)+\lambda c^{\alpha, S(n-1)}(n) \\
(\alpha+\lambda+\mu) c^{\alpha, S(n)}(n-1) & =c \mu+\mu c^{\alpha, S(n)}(n-2)+\lambda c^{\alpha, S(n)}(n) \\
(\alpha+\lambda) c^{\alpha, S(n)}(n) & =(c+r \lambda) \mu+\mu c^{\alpha, S(n)}(n-1) .
\end{aligned}
$$

From these, (46), (48), and Lemma 5.8(b), we obtain

$$
c^{\alpha, S(n)}(n)=c^{\alpha, S(n-1)}(n)+\mu \frac{(\alpha+\lambda+\mu) q^{\alpha}(n)-\mu}{(\alpha+\lambda)(\alpha+\lambda+\mu) q^{\alpha}(n)-\lambda \mu} c^{\alpha, S(n-1)}(n-1),
$$

as required. This completes the proof.

\subsubsection{Discounted MPI}

We next set out to establish $\operatorname{PCL}(\mathscr{F})$-indexability, and to calculate the discounted MPI. We start by constructing index $\nu^{\alpha, *}(i) \triangleq c^{\alpha, S(i)}(i) / w^{\alpha, S(i)}(i)$ (cf. (11)).

Proposition 5.15 Index $\nu^{\alpha, *}(i)$ is calculated by the following recursion:

$$
\nu^{\alpha, *}(i)= \begin{cases}\frac{c \mu}{\alpha}\left\{1-\left(\frac{\lambda}{\alpha+\lambda}\right)^{n}\right\}+r \mu\left(\frac{\lambda}{\alpha+\lambda}\right)^{n} & \text { if } i=1 \\ \nu^{\alpha, *}(i-1)-\frac{\nu^{\alpha, *}(i-1)-c^{\alpha, S(i-1)}(i)}{w^{\alpha, S(i)}(i)} & \text { if } 2 \leq i \leq n .\end{cases}
$$

Proof. We have, using $w^{\alpha, S(1)}(1)=1$ and (43),

$$
\nu^{\alpha, *}(1)=\frac{c^{\alpha, S(1)}(1)}{w^{\alpha, S(1)}(1)}=\frac{c \mu}{\alpha}\left\{1-\left(\frac{\lambda}{\alpha+\lambda}\right)^{n}\right\}+r \mu\left(\frac{\lambda}{\alpha+\lambda}\right)^{n} .
$$

Further, for $2 \leq i \leq n$, using Lemmas 5.9 and 5.14, we obtain

$$
\begin{aligned}
\nu^{\alpha, *}(i) & =\frac{c^{\alpha, S(i)}(i)}{w^{\alpha, S(i)}(i)} \\
& =\frac{c^{\alpha, S(i-1)}(i)+\mu \frac{(\alpha+\lambda+\mu) q^{\alpha}(i)-\mu}{(\alpha+\lambda)(\alpha+\lambda+\mu) q^{\alpha}(i)-\lambda \mu} c^{\alpha, S(i-1)}(i-1)}{w^{\alpha, S(i)}(i)} \\
& =\frac{c^{\alpha, S(i-1)}(i)+\mu \frac{(\alpha+\lambda+\mu) q^{\alpha}(i)-\mu}{(\alpha+\lambda)(\alpha+\lambda+\mu) q^{\alpha}(i)-\lambda \mu} w^{\alpha, S(i-1)}(i-1) \nu^{\alpha, *}(i-1)}{w^{\alpha, S(i)}(i)} \\
& =\frac{c^{\alpha, S(i-1)}(i)+\left\{w^{\alpha, S(i)}(i)-1\right\} \nu^{\alpha, *}(i-1)}{w^{\alpha, S(i)}(i)} \\
& =\nu^{\alpha, *}(i-1)-\frac{\nu^{\alpha, *}(i-1)-c^{\alpha, S(i-1)}(i)}{w^{\alpha, S(i)}(i)}
\end{aligned}
$$

as required. This completes the proof. 
Proposition 5.16 The following inequalities hold (strictly iff $c>\alpha r$ ):

(a) $\nu^{\alpha, *}(i-1) \geq c^{\alpha, S(i-1)}(i), \quad 2 \leq i \leq n$.

(b) $\nu^{\alpha, *}(1) \geq \nu^{\alpha, *}(2) \geq \cdots \geq \nu^{\alpha, *}(n)$.

Proof. We argue by upward induction on $i$. The case $i=2$ follows from

$$
\nu^{\alpha, *}(1)-c^{\alpha, S(1)}(2)=\left(\frac{\lambda}{\alpha+\lambda}\right)^{n-1} \frac{c-\alpha r}{\alpha+\lambda} \mu \geq 0 .
$$

Suppose now $\nu^{\alpha, S(i-1)}(i-1) \geq c^{\alpha, S(i-1)}(i)$ for some $2 \leq i \leq n-1$. Then, we can write

$$
\begin{aligned}
\nu^{\alpha, *}(i) & =c^{\alpha, S(i-1)}(i)+\frac{w^{\alpha, S(i)}(i)-1}{w^{\alpha, S(i)}(i)}\left\{\nu^{\alpha, *}(i-1)-c^{\alpha, S(i-1)}(i)\right\} \\
& \geq c^{\alpha, S(i-1)}(i) \geq c^{\alpha, S(i)}(i+1),
\end{aligned}
$$

where we have used the stated reformulation of (49), the induction hypothesis and the fact that $c^{\alpha, S(i-1)}(i)$ is nondecreasing in $i$, which follows immediately from (45). This completes the induction.

(b) This part follows directly from part (a) and Proposition 5.15.

In both parts it is readily seen that the inequalities are strict iff $c>\alpha r$. This completes the proof.

We can now give the main result of this section.

\section{Theorem 5.17}

(a) An $\alpha$-discount delay-sensitive class is PCL( $\mathscr{F})$-indexable, with MPI $\nu^{\alpha, *}(i)$.

(b) The class is $\alpha$-discount PCL( $\mathscr{F})$-indexable for any $\alpha>0$ iff it is pure delay-sensitive $(c>0=r)$.

Proof. (a) This part follows from Proposition 5.12(a) and Proposition 5.16(b).

(b) This part follows immediately from the above.

It is insightful to consider the limit of discounted MPI $\nu^{\alpha, *}(i)$ as the buffer size $n$ grows to infinity, for fixed $i$. In the pure delay-sensitive case, it is also of interest to consider the myopic index defined by

$$
\nu^{\text {myopic }}(i) \triangleq \lim _{\alpha \rightarrow \infty} \alpha \nu^{\alpha, *}(i) .
$$

The following result gives simple expressions for both limiting indices, which show corresponding asymptotic relations with the $c \mu$ rule.

\section{Proposition 5.18}

(a) $\lim _{n \rightarrow \infty} \nu^{\alpha, *}(i)=c \mu / \alpha, \quad i \geq 1$.

(b) $\nu^{\text {myopic }}(i)=c \mu, \quad 1 \leq i \leq n$.

Proof. Both parts follow immediately by taking the corresponding limits in Proposition 5.15(a).

\subsection{Bias criterion and MPI}

In order to design appropriate indices for delay-sensitive classes in average-criterion scheduling problem (3), we draw on the above analysis via a vanishing discount approach to define the index

$$
\nu^{*}(i) \triangleq \lim _{\alpha \searrow 0} \nu^{\alpha, *}(i), \quad 1 \leq i \leq n .
$$

We will show next that index $\nu^{*}(i)$ is well defined, and will derive it in closed form. We will further demonstrate that $\nu^{*}(i)$ is indeed an MPI, relative to the bias criterion, as introduced in Section 3.3.

To calculate $\nu^{*}(i)$ we will draw on recursion (49) in Proposition 5.15, which characterizes the discounted MPI $\nu^{\alpha, *}(i)$, letting the discount factor $\alpha$ vanish. We will thus need to calculate the undiscounted pivot marginal workloads

$$
w^{S(i)}(i) \triangleq \lim _{\alpha \searrow 0} w^{\alpha, S(i)}(i), \quad 1 \leq i \leq n,
$$


along with the undiscounted auxiliary marginal costs

$$
c^{S(i-1)}(i) \triangleq \lim _{\alpha \searrow 0} c^{\alpha, S(i-1)}(i), \quad 2 \leq i \leq n .
$$

The following result gives closed-form expressions for the $w^{S(i)}(i)$ 's. Notice that we write $\rho \triangleq \lambda / \mu$.

\section{Lemma 5.19}

(a) Terms $w^{S(i)}(i)$ are characterized by the recursion $w^{S(1)}(1)=1$ and

$$
w^{S(i)}(i)=1+\frac{w^{S(i-1)}(i-1)}{\rho}, \quad 2 \leq i \leq n .
$$

(b) The solution to such recursion is: for $1 \leq i \leq n$,

$$
w^{S(i)}(i)=\sum_{j=0}^{i-1} \rho^{-j}= \begin{cases}\frac{1-\rho^{i}}{(1-\rho) \rho^{i-1}} & \text { if } \rho \neq 1 \\ i & \text { if } \rho=1 .\end{cases}
$$

Proof. (a) The identity $w^{S(1)}(1)=1$ is trivial. Further, letting $q(i) \triangleq \lim _{\alpha \searrow 0} q^{\alpha}(i)$, we obtain from the first identity in Lemma 5.9 that

$$
w^{S(i)}(i)=1+\mu \frac{(\lambda+\mu) q(i)-\mu}{\lambda(\lambda+\mu) q(i)-\lambda \mu} w^{S(i-1)}(i-1)=1+\frac{w^{S(i-1)}(i-1)}{\rho}, \quad 2 \leq i \leq n,
$$

as required.

(b) This part follows immediately from part (a).

We next calculate the required undiscounted auxiliary marginal costs.

Lemma 5.20

$$
c^{S(i-1)}(i)=c \frac{n-i+1}{\rho}+r \mu, \quad 2 \leq i \leq n .
$$

Proof. We take limits in (45), using L'Hôpital's rule, to obtain

$$
\begin{aligned}
c^{S(i-1)}(i) & =\lim _{\alpha \searrow 0} c^{\alpha, S(i-1)}(i) \\
& =\lim _{\alpha \searrow 0} \frac{c \mu}{\alpha}\left\{1-\left(\frac{\lambda}{\alpha+\lambda}\right)^{n-i+1}\right\}+r \mu\left(\frac{\lambda}{\alpha+\lambda}\right)^{n-i+1} \\
& =c \frac{n-i+1}{\rho}+r \mu,
\end{aligned}
$$

as required.

We are now ready to calculate index $\nu^{*}(i)$.

\section{Proposition 5.21}

(a) Index $\nu^{*}(i)$ is calculated by the following recursion:

$$
\nu^{*}(i)= \begin{cases}c \frac{n}{\rho}+r \mu & \text { if } i=1 \\ \nu^{*}(i-1)-\frac{\nu^{*}(i-1)-c^{S(i-1)}(i)}{w^{S(i)}(i)} & \text { if } 2 \leq i \leq n .\end{cases}
$$



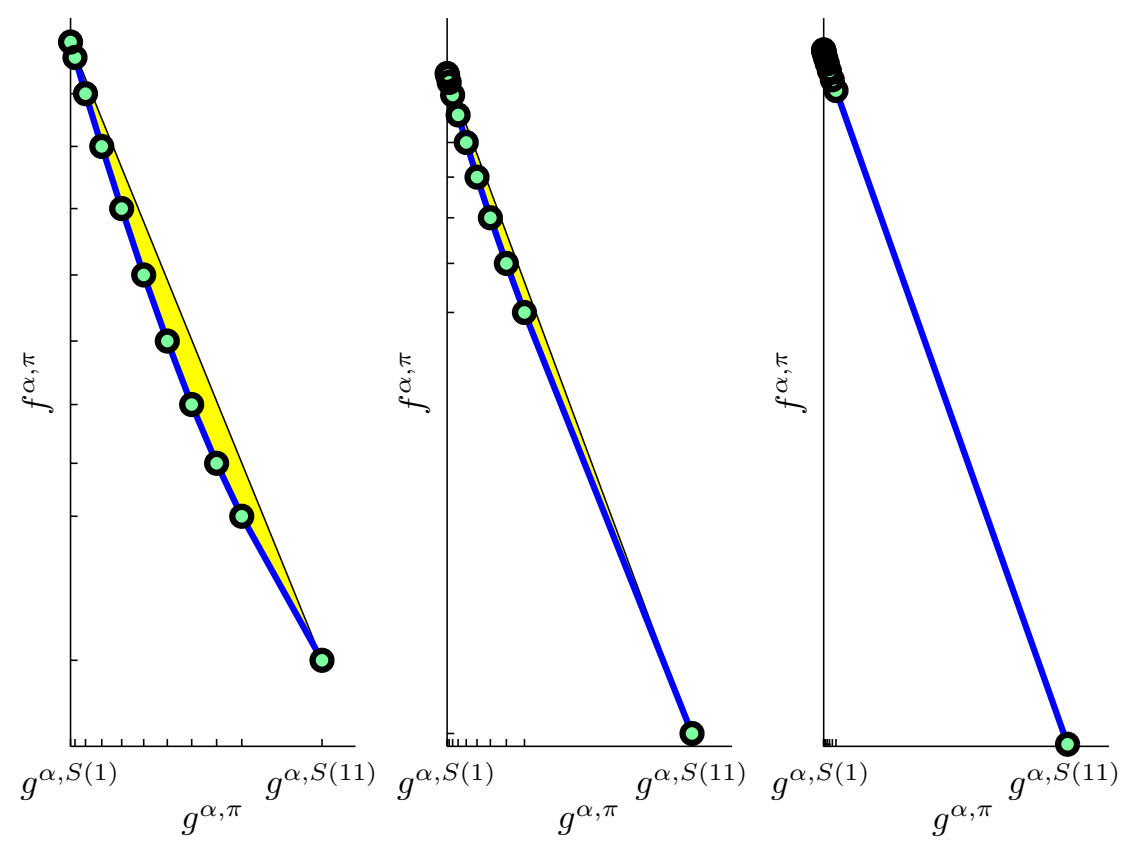

Figure 5: Discounted work-cost achievable performance region: $n=10, \lambda=0.9, \mu=1, r=4, c=5$, $\alpha=0.1,0.01,0.001$.

(b) The solution to such recursion is: for $1 \leq i \leq n$,

$$
\nu^{*}(i)= \begin{cases}\frac{c}{\rho}\left\{n-\frac{\rho}{1-\rho}+i \frac{\rho^{i}}{1-\rho^{i}}\right\}+r \mu & \text { if } \rho \neq 1 \\ c\left\{n-\frac{i-1}{2}\right\}+r \mu & \text { if } \rho=1 .\end{cases}
$$

Proof. (a) We have, taking the limit $\alpha \searrow 0$ in the first identity in (49),

$$
\begin{aligned}
\nu^{*}(1) & =\lim _{\alpha \searrow 0} \nu^{\alpha, *}(1) \\
& =\frac{c \mu}{\alpha}\left\{1-\left(\frac{\lambda}{\alpha+\lambda}\right)^{n}\right\}+r \mu\left(\frac{\lambda}{\alpha+\lambda}\right)^{n} \\
& =c \frac{n}{\rho}+r \mu .
\end{aligned}
$$

Further, the stated recursion follows immediately from its counterpart in (49).

(b) The result is readily verified by induction.

Having calculated index $\nu^{*}(i)$, we next argue that it is indeed an MPI, though not relative to the conventional average criterion in Section 3.2. To gain insight, let us start by understanding how the discounted work-cost achievable performance region changes as the discount factor vanishes. Figure 5 displays such region (appropriately scaled) in a specific instance under the discount factor values $\alpha=0.1,0.01,0.001$. Figure 5 illustrates the phenomenon which occurs in the general delay-sensitive case: as $\alpha$ approaches 0 , the achievable performance region of $\alpha$-scaled discounted work-cost pairs $\left(\alpha g^{\alpha, \pi}, \alpha f^{\alpha, \pi}\right)$, spanned under all admissible policies $\pi \in \Pi$, collapses into a line segment, as shown in Figure 6. The latter is precisely the average work-cost achievable performance region, spanned by average work-cost pairs $\left(g^{\pi}, f^{\pi}\right)$. The segment's right end-point is achieved by the $S(n+1)$-active policy (work whenever there are jobs in the queue), whereas its left end-point is achieved, not only by the $S(i)$-active policy, for $i=1, \ldots, n$, but by any policy which idles the server when the buffer is full. Notice that any such policy induces a Markov chain with absorbing state $n$, while the other states are transient. 


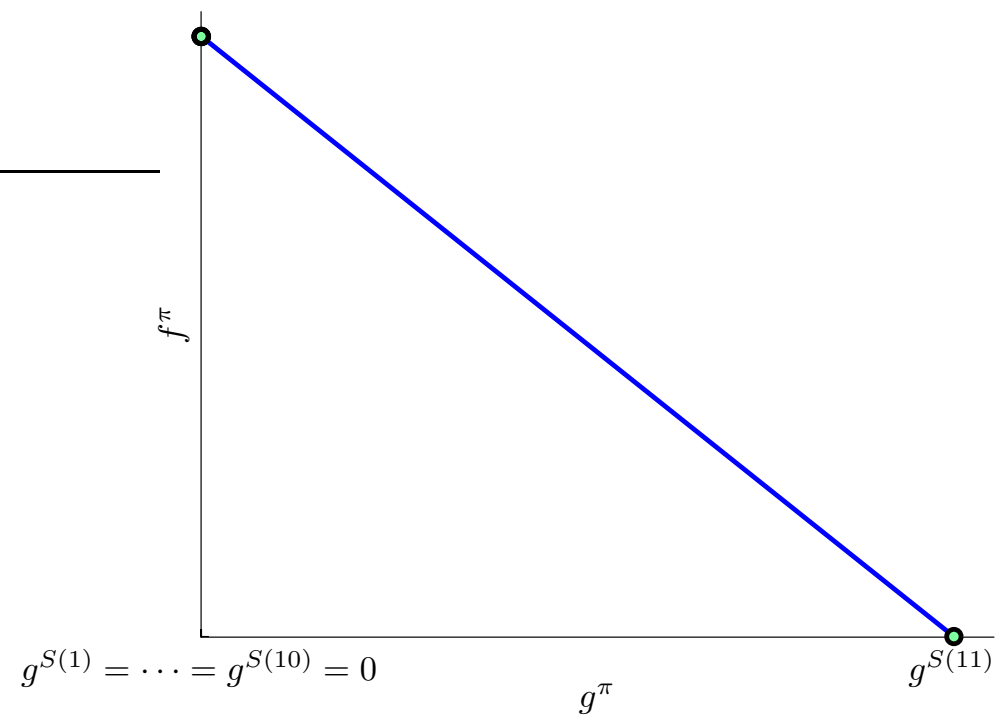

Figure 6: Average work-cost achievable performance region: $n=10, \lambda=0.9, \mu=1, r=4, c=5$.

We thus see that the required regularity condition (14) of average work measure $g^{\pi}$ relative to $\mathscr{F}$-policies does not hold. Therefore, the corresponding bandit is not average $\mathscr{F}$-indexable and the average MPI does not exist (cf. Definition 3.5). In light of the insight furnished by Figure 6, it is clear that average $\nu$-wage problem (13) is solved by the $S(n+1)$-active policy iff $\nu \leq \nu^{*}(n)$, whereas it is solved by any policy which idles the server in state $n$ iff $\nu \geq \nu^{*}(n)$.

To resolve such difficulty, we propose to use the concepts of bias $\mathscr{F}$-indexability and bias MPI, introduced in Section 3.3. The next result states that $\nu^{*}(i)$ is indeed the MPI in such setting.

Theorem 5.22 A delay-sensitive class $(c>0)$ is bias $\mathscr{F}$-indexable, with bias $M P I \nu^{*}(i)$.

Since our proof of Theorem 5.22 is based on new techniques, which draw on our PCL-indexability theory, we next outline the proof's main ingredients before presenting it. The key tool will be the Workload Reformulation lemma in Niño-Mora [10, Lemma 4.8]. This reformulates the difference between the discounted $\nu$-wage problem (4)'s objective

$$
v^{\alpha, \pi}(\nu) \triangleq f^{\alpha, \pi}+\nu g^{\alpha, \pi}
$$

under an arbitrary policy $\pi \in \Pi$ and under a given $\mathscr{F}$-policy, as a weighted sum of workload terms. Recall that we assume the initial state to be drawn from an arbitrary distribution — which is implicit in the notationassigning a positive probability to every state.

To state and deploy the result, we must introduce additional notation, as follows. For a policy $\pi \in \Pi$, action $a \in\{0,1\}$ and state $j \in N$, we define $x^{\alpha, a, \pi}(j)$ as the discounted state-action occupancy measure, i.e. the expected total discounted time that action $a$ is taken in state $j$ under policy $\pi$. We further define, for an active-state set $S \in \mathscr{F}$, the aggregate marginal work measures

$$
W^{\alpha, S, 0, \pi} \triangleq \sum_{j \in S} w^{\alpha, S}(j) x^{\alpha, 0, \pi}(j), \quad W^{\alpha, S, 1, \pi} \triangleq \sum_{j \in N\{0,1\} \backslash S} w^{\alpha, S}(j) x^{\alpha, 1, \pi}(j) .
$$

We next state the Workload Reformulation lemma, as it applies to the present model.

Lemma 5.23 For any state $2 \leq i \leq n$, policy $\pi \in \Pi$ and wage $\nu \in \mathbb{R}$, the discounted $\nu$-wage problem's objective can be reformulated as

$$
\begin{aligned}
v^{\alpha, \pi}(\nu)= & v^{\alpha, S(i)}(\nu)+W^{\alpha, S(i), 1, \pi}\left\{\nu-\nu^{\alpha, *}(i)\right\}+W^{\alpha, S(i), 0, \pi}\left\{\nu^{\alpha, *}(i-1)-\nu\right\} \\
& +\sum_{j=1}^{i-2} W^{\alpha, S(j+1), 0, \pi}\left\{\nu^{\alpha, *}(j)-\nu^{\alpha, *}(j+1)\right\}+\sum_{j=i+1}^{n} W^{\alpha, S(j), 1, \pi}\left\{\nu^{\alpha, *}(j-1)-\nu^{\alpha, *}(j)\right\} .
\end{aligned}
$$


We will further use the fact that the undiscounted marginal workloads

$$
w^{S}(i) \triangleq \lim _{\alpha \searrow 0} w^{\alpha, S}(i), \quad 1 \leq i \leq n, S \in \mathscr{F},
$$

are well defined and positive. This follows immediately by setting $\alpha=0$ in Figure 3's recursion.

Another ingredient in the proof is the observation that, under any admissible policy $\pi$ which idles the server in state $n$, the following limiting occupancy measures are finite:

$$
\begin{aligned}
& x^{1, \pi}(j) \triangleq \lim _{\alpha \searrow 0} x^{\alpha, 1, \pi}(j)<\infty, \quad 1 \leq j \leq n \\
& x^{0, \pi}(j) \triangleq \lim _{\alpha \searrow 0} x^{\alpha, 0, \pi}(j)<\infty, \quad 1 \leq j \leq n-1 .
\end{aligned}
$$

Such result is an immediate consequence of the fact that under any such policy state $n$ is absorbing.

It follows readily from the above that, under any such policy $\pi$, the following limiting aggregate marginal workloads are finite:

$$
\begin{aligned}
& W^{S, 0, \pi} \triangleq \sum_{j \in S} w^{S}(j) x^{0, \pi}(j)=\lim _{\alpha \searrow 0} W^{\alpha, S, 0, \pi}<\infty, \quad n \notin S \in \mathscr{F} \\
& W^{S, 1, \pi} \triangleq \sum_{j \in N\{0,1\} \backslash S} w^{S}(j) x^{1, \pi}(j)=\lim _{\alpha \searrow 0} W^{\alpha, S, 1, \pi}<\infty, \quad S \in \mathscr{F} .
\end{aligned}
$$

We next draw on the above to present a counterpart to Lemma 5.23 well-suited to establish bias optimality.

Lemma 5.24 For any state $2 \leq i \leq n$, wage $\nu \in \mathbb{R}$ and admissible policy $\pi$ which idles the server in state $n$, it holds that:

$$
\begin{aligned}
\lim _{\alpha \searrow 0} v^{\alpha, \pi}(\nu)-v^{\alpha, S(i)}(\nu)= & W^{S(i), 1, \pi}\left\{\nu-\nu^{*}(i)\right\}+W^{S(i), 0, \pi}\left\{\nu^{*}(i-1)-\nu\right\} \\
& +\sum_{j=1}^{i-2} W^{S(j+1), 0, \pi}\left\{\nu^{*}(j)-\nu^{*}(j+1)\right\}+\sum_{j=i+1}^{n} W^{S(j), 1, \pi}\left\{\nu^{*}(j-1)-\nu^{*}(j)\right\}
\end{aligned}
$$

Proof. We use Lemma 5.23, (50), and (51)-(53) to write, for any such policy $\pi$,

$$
\begin{aligned}
v^{\alpha, \pi}(\nu)-v^{\alpha, S(i)}(\nu)= & W^{\alpha, S(i), 1, \pi}\left\{\nu-\nu^{\alpha, *}(i)\right\}+W^{\alpha, S(i), 0, \pi}\left\{\nu^{\alpha, *}(i-1)-\nu\right\} \\
& +\sum_{j=1}^{i-2} W^{\alpha, S(j+1), 0, \pi}\left\{\nu^{\alpha, *}(j)-\nu^{\alpha, *}(j+1)\right\}+\sum_{j=i+1}^{n} W^{\alpha, S(j), 1, \pi}\left\{\nu^{\alpha, *}(j-1)-\nu^{\alpha, *}(j)\right\} \\
= & W^{S(i), 1, \pi}\left\{\nu-\nu^{*}(i)\right\}+W^{S(i), 0, \pi}\left\{\nu^{*}(i-1)-\nu\right\} \\
& +\sum_{j=1}^{i-2} W^{S(j+1), 0, \pi}\left\{\nu^{*}(j)-\nu^{*}(j+1)\right\}+\sum_{j=i+1}^{n} W^{S(j), 1, \pi}\left\{\nu^{*}(j-1)-\nu^{*}(j)\right. \\
& +o(1) \text { as } \alpha \searrow 0,
\end{aligned}
$$

as required.

We are now ready to give the proof of Theorem 5.22. See Definition 3.8 and (19).

Proof of Theorem 5.22. Let $2 \leq i \leq n$, and suppose $\nu \in\left[\nu^{*}(i), \nu^{*}(i-1)\right]$. Let $\pi$ be a stationary policy which idles the server in state $n$. Then, we use Lemma 5.24 to obtain

$$
\begin{aligned}
\lim _{\alpha \searrow 0} v^{\alpha, \pi}(\nu)-v^{\alpha, S(i)}(\nu)= & W^{S(i), 1, \pi}\left\{\nu-\nu^{*}(i)\right\}+W^{S(i), 0, \pi}\left\{\nu^{*}(i-1)-\nu\right\} \\
& +\sum_{j=1}^{i-2} W^{S(j+1), 0, \pi}\left\{\nu^{*}(j)-\nu^{*}(j+1)\right\}+\sum_{j=i+1}^{n} W^{S(j), 1, \pi}\left\{\nu^{*}(j-1)-\nu^{*}(j)\right\} \\
\geq & 0,
\end{aligned}
$$


since every term in the right-hand side of (54) is nonnegative. It thus follows that the $S(i)$-active policy is bias optimal for the $\nu$-wage problem.

Conversely, suppose the $S(i)$-active policy is bias optimal for the $\nu$-wage problem. Then, taking $\pi=$ $S(i+1)$ in Lemma 5.24, we obtain

$$
0 \leq \lim _{\alpha \searrow 0} v^{\alpha, S(i+1)}(\nu)-v^{\alpha, S(i)}(\nu)=w^{S(i)}(i) x^{1, S(i+1)}(i)\left\{\nu-\nu^{*}(i)\right\}
$$

Since $w^{S(i)}(i) x^{1, S(i+1)}(i)>0$, it follows that $\nu \geq \nu^{*}(i)$.

Further, taking $\pi=S(i-1)$ in 5.24, we obtain

$$
0 \leq \lim _{\alpha \searrow 0} v^{\alpha, S(i-1)}(\nu)-v^{\alpha, S(i)}(\nu)=w^{S(i)}(i-1) x^{0, S(i-1)}(i-1)\left\{\nu^{*}(i-1)-\nu\right\} .
$$

Since $w^{S(i)}(i-1) x^{0, S(i-1)}(i-1)>0$, it follows that $\nu^{*}(i-1) \geq \nu$. This completes the proof.

\subsection{Relation with the $c \mu$-rule}

Since the optimal policy for the infinite-buffer delay-sensitive version of scheduling problems (2) and (3) is the classical $c \mu$-rule, it is insightful to investigate the behaviour of the MPI policies as buffer sizes grow.

Under the discounted criterion, Proposition 5.18(a) shows that the MPI policy does approach asymptotically the $c \mu$ rule as buffer sizes grow.

The relation is not as straightforward under the average criterion. However, the bias MPI expression in Proposition 5.21 reveals that the MPI policy will asymptotically approach the $c \mu$-rule provided the following condition holds:

$$
\frac{n_{1}}{\lambda_{1}} \approx \frac{n_{2}}{\lambda_{2}} \approx \cdots \approx \frac{n_{K}}{\lambda_{K}}, \quad \text { as } n_{1}, \ldots, n_{K} \rightarrow \infty
$$

i.e. provided buffer sizes grow in fixed proportion to arrival rates.

\section{Computational study}

We next discuss the results of a computational study on the performance of MPI policies across the range of 32 two-class instances shown in Table 1. As shown in Figure 1, in some instances we vary the discount factor $\alpha$, where the value $\alpha=0$ represents the average criterion. The results are presented in Table 2. For each instance, we report the optimal cost $f^{*}$, the cost $f^{\mathrm{MPI}}$ under the corresponding MPI policy, and the cost $f^{\text {naive }}$ under a naive policy. In the discounted case, such costs correspond to the discounted cost measure $f^{\alpha, \pi}$ scaled by $\alpha$, for consistency with the limiting average case as $\alpha$ vanishes. The naive policy uses the index $\left(c_{k}+r_{k}\right) \mu_{k}$ for a delay-sensitive class $k$, and it uses the static index $r_{k} \mu_{k}$ for a loss-sensitive class, breaking ties with the dynamic index $r_{k} \mu_{k}\left(n_{k}-L_{k}(t)\right)$. The results have been obtained by solving the corresponding dynamic programming or evaluation equations, via a Matlab implementation by the author.

Notice that instances 1-9 are pure loss-sensitive, instances 10-21 are pure delay-sensitive, and instances 22-32 are mixed. Further, instances are based on the example in Figure 1 of Kim and Van Oyen [4].

The results reported in Table 2 show that the MPI policy is near-optimal in every instance, and often even optimal, and it significantly outperforms the naive policy in most instances. Only in instances 21 and 29 does the naive policy slightly outperform the MPI policy.

We have further compared for every instance the structure of the optimal policy with that of the MPI and the naive policies. We have found that in instances where both classes are delay-sensitive, the optimal policy is consistent with the MPI policy in that both are represented by an switching curve which is nondecreasing in the queue lengths $\left(i_{1}, i_{2}\right)$. See, e.g. Figure 7 , which displays the structure of the optimal, the MPI and the naive policy for instance 8 , where a square box means that queue 1 is served, and a star means that queue 2 is served.

In instances where both classes are delay-sensitive, however, one might be tempted to conjecture that optimal policies are consistent with the state orderings induced by MPI policies. However, such is not the case in general, as illustrated by Figure 8 on instance 14. 


\begin{tabular}{|c|c|c|c|c|c|c|}
\hline Instance & $\bar{n}$ & $\lambda$ & $\mu$ & c & $\mathbf{r}$ & $\alpha$ \\
\hline 1 & $(1,1)$ & $(0.8,0.5)$ & $(1,1.2)$ & $(0,0)$ & $(1,2)$ & 0.5 \\
\hline 2 & $(10,10)$ & $(0.8,0.5)$ & $(1,1.2)$ & $(0,0)$ & $(1,2)$ & 0.5 \\
\hline 3 & $(10,10)$ & $(0.8,0.5)$ & $(1,1.2)$ & $(0,0)$ & $(1,2)$ & 0.1 \\
\hline 4 & $(10,10)$ & $(0.8,0.5)$ & $(1,1.2)$ & $(0,0)$ & $(1,2)$ & 0.01 \\
\hline 5 & $(10,10)$ & $(0.8,0.5)$ & $(1,1.2)$ & $(0,0)$ & $(1,2)$ & 0 \\
\hline 6 & $(10,10)$ & $(0.8,0.5)$ & $(1,2)$ & $(0,0)$ & $(1,0.5)$ & 0.5 \\
\hline 7 & $(10,10)$ & $(0.8,0.5)$ & $(1,2)$ & $(0,0)$ & $(1,0.5)$ & 0.1 \\
\hline 8 & $(10,10)$ & $(0.8,0.5)$ & $(1,2)$ & $(0,0)$ & $(1,0.5)$ & 0.01 \\
\hline 9 & $(10,10)$ & $(0.8,0.5)$ & $(1,2)$ & $(0,0)$ & $(1,0.5)$ & 0 \\
\hline 10 & $(5,5)$ & $(1,0.4)$ & $(2,1)$ & $(1.1,2)$ & $(0,0)$ & 0.5 \\
\hline 11 & $(5,5)$ & $(1,0.4)$ & $(2,1)$ & $(1.1,2)$ & $(0,0)$ & 0.1 \\
\hline 12 & $(5,5)$ & $(1,0.4)$ & $(2,1)$ & $(1.1,2)$ & $(0,0)$ & 0.01 \\
\hline 13 & $(5,5)$ & $(1,0.4)$ & $(2,1)$ & $(1.1,2)$ & $(0,0)$ & 0 \\
\hline 14 & $(10,10)$ & $(1,0.4)$ & $(2,1)$ & $(1.1,2)$ & $(0,0)$ & 0.5 \\
\hline 15 & $(10,10)$ & $(1,0.4)$ & $(2,1)$ & $(1.1,2)$ & $(0,0)$ & 0.1 \\
\hline 16 & $(10,10)$ & $(1,0.4)$ & $(2,1)$ & $(1.1,2)$ & $(0,0)$ & 0.01 \\
\hline 17 & $(10,10)$ & $(1,0.4)$ & $(2,1)$ & $(1.1,2)$ & $(0,0)$ & 0 \\
\hline 18 & $(10,10)$ & $(0.3,0.1)$ & $(1,1)$ & $(1.1,1)$ & $(0,0)$ & 0.5 \\
\hline 19 & $(10,10)$ & $(0.3,0.1)$ & $(1,1)$ & $(1.1,1)$ & $(0,0)$ & 0.1 \\
\hline 20 & $(10,10)$ & $(0.3,0.1)$ & $(1,1)$ & $(1.1,1)$ & $(0,0)$ & 0.01 \\
\hline 21 & $(10,10)$ & $(0.3,0.1)$ & $(1,1)$ & $(1.1,1)$ & $(0,0)$ & 0 \\
\hline 22 & $(10,10)$ & $(0.8,0.3)$ & $(1,2)$ & $(0,0.4)$ & $(1,0)$ & 0.5 \\
\hline 23 & $(10,10)$ & $(0.8,0.3)$ & $(1,2)$ & $(0,0.4)$ & $(1,0)$ & 0.1 \\
\hline 24 & $(10,10)$ & $(0.8,0.3)$ & $(1,2)$ & $(0,0.4)$ & $(1,0)$ & 0.01 \\
\hline 25 & $(10,10)$ & $(0.8,0.3)$ & $(1,2)$ & $(0,0.4)$ & $(1,0)$ & 0 \\
\hline 26 & $(10,10)$ & $(0.3,0.7)$ & $(2,2)$ & $(1.01,1)$ & $(10,10)$ & 1 \\
\hline 27 & $(10,10)$ & $(0.3,0.7)$ & $(2,2)$ & $(1.01,1)$ & $(10,10)$ & 0.5 \\
\hline 28 & $(10,10)$ & $(0.3,0.7)$ & $(2,2)$ & $(1.01,1)$ & $(10,10)$ & 0.2 \\
\hline 29 & $(10,10)$ & $(0.3,0.7)$ & $(2,2)$ & $(1.01,1)$ & $(10,10)$ & 0.1 \\
\hline 30 & $(10,10)$ & $(0.3,0.7)$ & $(2,2)$ & $(1.01,1)$ & $(10,10)$ & 0.05 \\
\hline 31 & $(10,10)$ & $(0.3,0.7)$ & $(2,2)$ & $(1.01,1)$ & $(10,10)$ & 0.01 \\
\hline 32 & $(10,10)$ & $(0.3,0.7)$ & $(2,2)$ & $(1.01,1)$ & $(10,10)$ & 0 \\
\hline
\end{tabular}

Table 1: Cases investigated.
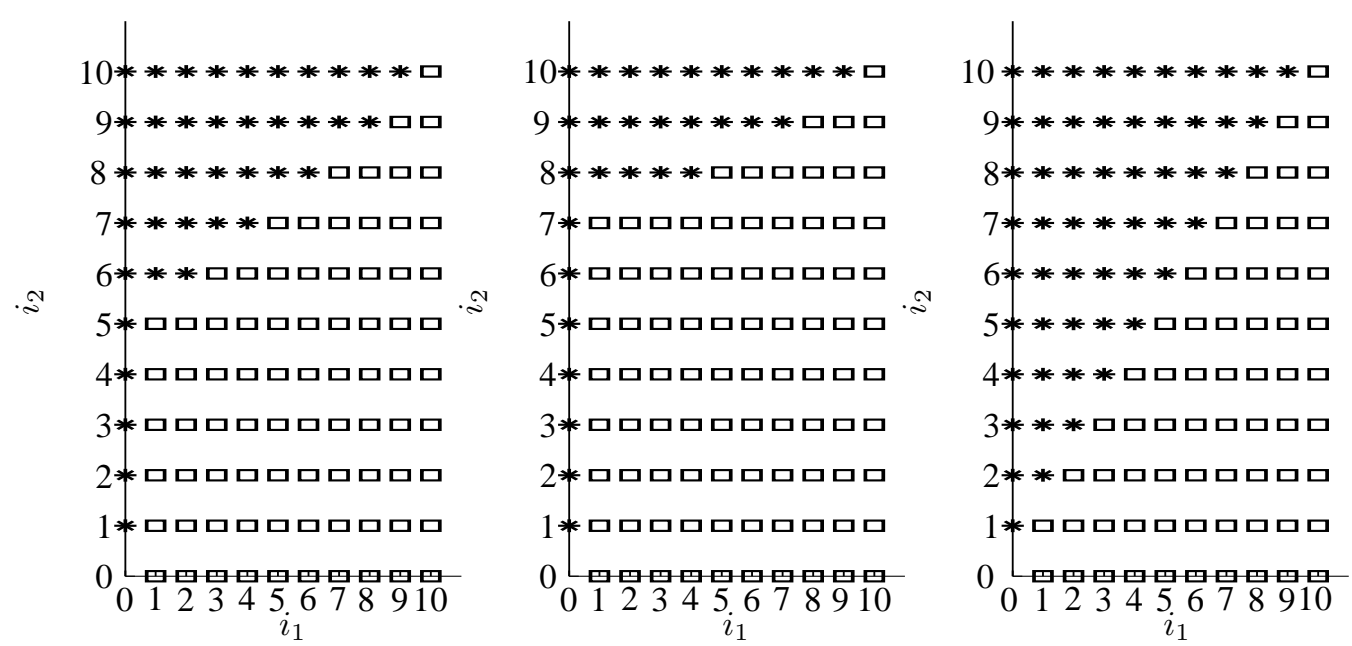

Figure 7: Instance 8: structure of optimal, MPI and naive policies. 


\begin{tabular}{|c|c|c|c|}
\hline Instance & $f^{*}$ & $f^{\mathrm{MPI}}$ & $f^{\text {naive }}$ \\
\hline 1 & 0.7844 & 0.7844 & 0.7844 \\
\hline 2 & 0.1211 & 0.1211 & 0.2007 \\
\hline 3 & 0.1338 & 0.1340 & 0.2643 \\
\hline 4 & 0.2083 & 0.2095 & 0.2481 \\
\hline 5 & 0.2273 & 0.2370 & 0.2370 \\
\hline 6 & 0.0728 & 0.0729 & 0.0731 \\
\hline 7 & 0.0690 & 0.0692 & 0.0703 \\
\hline 8 & 0.0817 & 0.0820 & 0.0825 \\
\hline 9 & 0.0870 & 0.0873 & 0.0875 \\
\hline 10 & 6.8987 & 6.9031 & 7.0784 \\
\hline 11 & 5.2968 & 5.2991 & 6.1185 \\
\hline 12 & 3.9894 & 3.9894 & 5.2446 \\
\hline 13 & 3.7763 & 3.7763 & 5.0812 \\
\hline 14 & 14.6226 & 14.6247 & 14.7759 \\
\hline 15 & 11.7361 & 11.7446 & 12.9384 \\
\hline 16 & 6.8651 & 6.8651 & 9.2980 \\
\hline 17 & 5.7525 & 5.7525 & 8.1041 \\
\hline 18 & 9.2155 & 9.2155 & 9.2314 \\
\hline 19 & 5.9517 & 5.9898 & 6.0382 \\
\hline 20 & 1.6257 & 1.6613 & 1.6667 \\
\hline 21 & 0.7095 & 0.7220 & 0.7095 \\
\hline 22 & 1.3088 & 1.3088 & 2.1302 \\
\hline 23 & 0.5757 & 0.5757 & 2.2043 \\
\hline 24 & 0.1872 & 0.1872 & 1.9814 \\
\hline 25 & 0.1288 & 0.1288 & 1.8475 \\
\hline 26 & 10.1600 & 10.1758 & 10.2461 \\
\hline 27 & 8.5420 & 8.5480 & 8.5572 \\
\hline 28 & 6.6019 & 6.6100 & 6.6112 \\
\hline 29 & 4.8911 & 5.0195 & 4.9462 \\
\hline 30 & 3.3978 & 3.4227 & 3.4227 \\
\hline 31 & 1.5761 & 1.5767 & 1.5767 \\
\hline 32 & 1.0014 & 1.0014 & 1.0014 \\
\hline
\end{tabular}

Table 2: Results of computational experiments.
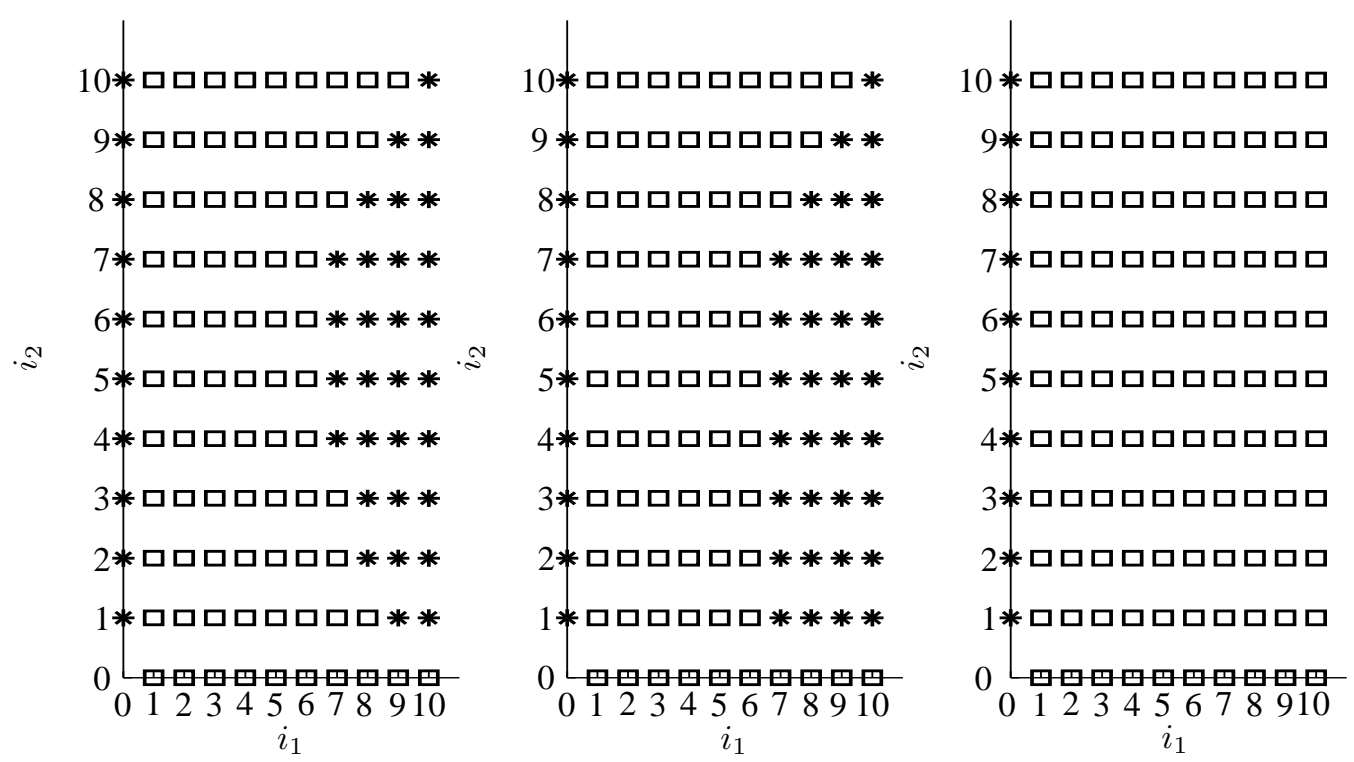

Figure 8: Instance 14: structure of optimal, MPI and naive policies. 


\section{Acknowledgment}

This research has been supported in part by the Spanish Ministry of Education and Science under a Ramón y Cajal Investigator Award and project MTM2004-02334, and by the European Union's Network of Excelence Euro-NGI. The author has presented preliminary versions of this paper at the 2nd Conference on Performance Modelling and Evaluation of Heterogeneous Networks, HET-NETs '04 (Ilkley, UK, July 26-28, 2004), the First Euro-NGI Workshop on Network Optimization and Control (INRIA-Sophia Antipolis, France, October 14-15, 2004), a Eurandom seminar (Eindhoven, November 16, 2004), and the First Euro-NGI Conference on Next Generation Internet Networks, NGI '05 (Rome, April 18-20, 2005). A short version of the paper appeared in the proceedings of the latter conference. See [9].

\section{References}

[1] D. Blackwell. Discrete dynamic programming. Ann. Math. Statist., 33:719-726, 1962.

[2] D. R. Cox and W. L. Smith. Queues. Methuen, London, 1961.

[3] J. C. Gittins. Bandit processes and dynamic allocation indices. J. Roy. Statist. Soc. Ser. B, 41:148-177, 1979. With discussion.

[4] E. Kim and M. P. Van Oyen. Beyond the $c \mu$ rule: Dynamic scheduling of a two-class loss queue. Math. Meth. Oper. Res., 48:17-36, 1998.

[5] M. E. Lewis and M. L. Puterman. Bias optimality. In E. Feinberg and A. Schwartz, editors, Handbook of Markov Decision Processes, pages 89-111. Kluwer, Boston, Massachusetts, 2002.

[6] R. A. Milito and H. Levy. Modeling and dynamic scheduling of a queueing system with blocking and starvation. IEEE Trans. Comm., 37:1318-1329, 1989.

[7] J. Niño-Mora. Restless bandits, partial conservation laws and indexability. Adv. in Appl. Probab., 33:76-98, 2001.

[8] J. Niño-Mora. Dynamic allocation indices for restless projects and queueing admission control: a polyhedral approach. Math. Program., 93:361-413, 2002.

[9] J. Niño-Mora. Marginal productivity index policies for scheduling multiclass delay-/loss-sensitive traffic. In Proceedings of the First Euro-NGI Conference on Next Generation Internet Networks (NGI 05), pages 61-66, 2005.

[10] J. Niño-Mora. Restless bandit marginal productivity indices, diminishing returns and optimal control of make-to-order/make-to-stock $M / G / 1$ queues. Math. Oper. Res. (in press), 2005. Online copy: http://halweb.uc3m.es/jnino/eng/jnmmor.pdf.

[11] M. L. Puterman. Markov Decision Processes: Discrete Stochastic Dynamic Programming. Wiley, New York, 1994.

[12] P. D. Sparaggis, C. G. Cassandras, and D. Towsley. On the duality between routing and scheduling systems with finite buffer space. IEEE Trans. Automat. Control, 38:1440-1446, 1993.

[13] K. M. Wasserman and N. Bambos. Optimal server allocation to parallel queues with finite-capacity buffers. Probab. Engr. Inf. Sci., 10:279-285, 1996.

[14] P. Whittle. Restless bandits: Activity allocation in a changing world. In J. Gani, editor, A Celebration of Applied Probability, J. Appl. Probab. Special Vol. 25A, pages 287-298. Applied Probability Trust, 1988. 